Groups Geom. Dyn. 8 (2014), 565-598

DOI $10.4171 / \mathrm{GGD} / 239$
Groups, Geometry, and Dynamics

(C) European Mathematical Society

\title{
On the geometry of the edge splitting complex
}

\author{
Lucas Sabalka and Dmytro Savchuk
}

\begin{abstract}
The group Out of outer automorphisms of the free group has been an object of active study for many years, yet its geometry is not well understood. Recently, effort has been focused on finding a hyperbolic complex on which Out acts, in analogy with the curve complex for the mapping class group. Here, we focus on one of these proposed analogues: the edge splitting complex $\mathcal{E} S_{n}$, equivalently known as the separating sphere complex. We characterize geodesic paths in its 1 -skeleton $\& S_{n}^{1}$ algebraically, and use our characterization to find lower bounds on distances between points in this graph.

Our distance calculations allow us to find quasiflats of arbitrary dimension in $\mathcal{E} \delta_{n}$. This shows that $\mathcal{E} \delta_{n}$ : is not hyperbolic, has infinite asymptotic dimension, and is such that every asymptotic cone is infinite dimensional. These quasiflats contain an unbounded orbit of a reducible element of Out. As a consequence, there is no coarsely Out-equivariant quasiisometry between $\mathcal{E} S_{n}$ and other proposed curve complex analogues, including the regular free splitting complex $\mathcal{F}_{n}$, the (nontrivial intersection) free factorization complex $\mathcal{F}_{\mathcal{F}}$, and the free factor complex $\mathscr{F}_{n}$.
\end{abstract}

Mathematics Subject Classification (2010). 20F65, $20 \mathrm{E} 36$.

Keywords. Outer automorphisms of free groups, curve complex, quasi-isometry, hyperbolicity, asymptotic dimension.

\section{Introduction}

Let $\operatorname{Out}\left(F_{n}\right)$ denote the group of outer automorphisms of the free group $F_{n}$ of rank $n$, where we assume throughout this paper that $n>2$. We wish to study the geometry of $\operatorname{Out}\left(F_{n}\right)$, by examining the geometry of certain spaces on which group acts. There is a strong analogy between $\operatorname{Out}\left(F_{n}\right)$ and the mapping class group of a surface on the one hand and arithmetic groups on the other, which has been pursued quite fruitfully in the last couple of decades. This approach began in earnest with the foundational paper of Culler and Vogtmann [9], which introduced Outer Space, the analogue for $\operatorname{Out}\left(F_{n}\right)$ of Teichmüller space for the mapping class group and of symmetric spaces for arithmetic groups. The work that followed has yielded numerous statements about the topological, homological, and cohomological properties of $\operatorname{Out}\left(F_{n}\right)$ and the spaces it acts upon - see for instance [29] for an excellent survey. 
While the topology of Outer Space is well understood, its geometry is not. In contrast, the geometries of Teichmüller space and the symmetric spaces are well studied. One key ingredient for the study of Teichmüller space is the celebrated result of Masur and Minsky, who proved that the curve complex is hyperbolic [25]. The curve complex is the complex whose vertex set is the set of isotopy classes of simple closed curves on the surface, and where a $k$-simplex corresponds to $k+1$ isotopy classes which have representatives that are disjoint. Moreover, there is a 'nice' map from Teichmüller space to the curve complex, so that the hyperbolicity of the curve complex has led to many further statements on the geometry of Teichmüller space and the mapping class group [3]. The curve complex has been used, for instance, to prove quasiisometric rigidity of the mapping class group. The analogous key ingredients in the study of arithmetic groups are Tits buildings, which again yield, for instance, rigidity theorems. The 'correct' analogue for $\operatorname{Out}\left(F_{n}\right)$ is still unknown, and much recent effort has been directed towards finding one - in particular, one which is hyperbolic.

There are many possible ways of defining such an analogue. We will formally define the most relevant two soon, but we leave definitions of the remaining complexes and graphs to the references. Before we list some of proposed analogues, let us mention that in most cases they are defined as complexes, but for our purposes (detecting hyperbolicity and distinguishing the spaces up to quasiisometry) it is enough to consider just 1-skeletons of the complexes. For each complex we will denote its 1 -skeleton by adding superscript ' 1 ' to the notation of the complex. Although we will rigorously define and work only with 1-skeletons of the complexes to simplify exposition, our results apply to the corresponding complexes as well.

Complexes and graphs which deserve mention as possible analogues include: the sphere complex [15], also called the free splitting complex $\mathcal{F} S_{n}$, and its 1-skeleton $\widetilde{F} S_{n}^{1}$, called the free splitting graph [1]; the (common refinement) free factorization complex, defined in [16] for $\operatorname{Aut}\left(F_{n}\right)$, whose $\operatorname{Out}\left(F_{n}\right)$ version we call the edge splitting complex $\mathcal{E} S_{n}$ in this paper; the free factor complex $\mathscr{F}_{n}$ (also defined initially for $\operatorname{Aut}\left(F_{n}\right)$ in [17]); and the intersection graph of Kapovich and Lustig [20]. Kapovich and Lustig [20] in fact list 9 graphs which could be an analogue of the curve complex. They include the 1-skeleton of the edge splitting complex which we call the edge splitting graph $\mathcal{E} S_{n}^{1}$ (called the free splitting graph in [20], though they do not allow $\mathrm{HNN}$-extensions as vertices) and the 1-skeleton of the free factor complex which we call the free factor graph $\mathscr{F}_{n}^{1}$ (called the dominance graph in [20]).

Kapovich and Lustig claim that, among the 9 graphs they list, there are at most 3 quasiisometry classes. Representatives of the three mentioned quasiisometry classes are the edge splitting graph, the free factor graph, and the intersection graph. We intend to show that the class containing the edge splitting graph cannot be coarsely $\operatorname{Out}\left(F_{n}\right)$-equivariantly quasiisometric to the free factor graph, which implies that it is not equivariantly quasiisometric to the intersection graph either (as there is an equivariant Lipschitz map from the factor graph to the intersection graph). For our purposes, it will be more convenient to use what we call the (nontrivial intersection) 
free factorization graph $\mathcal{F} \mathcal{F}_{n}^{1}$ instead of the free factor graph as a representative of the second quasiisometry class. Note that our free factorization graph is not the 1-skeleton of Hatcher and Vogtmann's (common refinement) free factorization complex (herein called the edge splitting graph), and that this graph was called the dual free splitting graph in [20], though again in the latter reference they did not allow HNN-extensions as vertices. We now define the edge splitting graph and the free factorization graph.

Definition 1.1 $\left(\mathcal{E} S_{n}^{1}\right.$ and $\left.\mathscr{F F}^{1}{ }_{n}^{1}\right)$. For $n>2$, define the edge splitting graph, denoted $\mathcal{E} S_{n}^{1}$, to be the graph whose vertices correspond to conjugacy classes $\left[\left\langle x_{1}, \ldots, x_{k}\right\rangle *\right.$ $\left.\left\langle x_{k+1}, \ldots, x_{n}\right\rangle\right]$ of free factorizations $\left\langle x_{1}, \ldots, x_{k}\right\rangle *\left\langle x_{k+1}, \ldots, x_{n}\right\rangle$ of $F_{n}$ into two nontrivial free factors. Two vertices of $\& S_{n}^{1}$ are connected with an edge if there exists a free factorization in each conjugacy class such that the two factorizations have a common refinement which is a free factorization into three nontrivial factors.

The (nontrivial intersection) free factorization graph $\mathcal{F F}_{\mathcal{F}}{ }_{n}^{1}$ has the same vertex set as $\mathcal{E} S_{n}^{1}$. Two vertices $[A * B]$ and $[C * D]$ are connected with an edge in $\mathcal{F F}{ }_{n}^{1}$ if one of $A \cap C, A \cap D, B \cap C$, or $B \cap D$ is nontrivial.

The name of the edge splitting graph comes from Bass-Serre theory, where such a free factorization is a graph of groups decomposition of $F_{n}$ with underlying graph having exactly two vertices and a single edge (with trivial edge group) between them. Note that the related free splitting graph $\mathscr{F} S_{n}^{1}$ (the 1-skeleton of the free splitting complex or equivalently the sphere complex) is defined similarly to $E S_{n}^{1}$, but also allows conjugacy classes of splittings of $F_{n}$ as $\mathrm{HNN}$-extensions as vertices.

There are alternate ways to define each of these objects. In particular, the edge splitting graph $\& S_{n}^{1}$ is also known as the separating sphere graph, whose vertices are homotopy classes of separating essential embedded spheres in a 3-manifold with fundamental group $F_{n}$, and two vertices are adjacent if they have disjoint representatives. The free factorization graph can equivalently be defined in terms of Bass-Serre theory, where vertices are Bass-Serre trees of free splittings up to $\operatorname{Out}\left(F_{n}\right)$-equivariant isometry, and adjacency corresponds to having a common elliptic element.

Note there is a natural action of $\operatorname{Out}\left(F_{n}\right)$ on all of these spaces, where for $\varepsilon S_{n}^{1}$ and $\mathcal{F}_{\mathcal{F}}{ }_{n}^{1}$ the action is induced by the action of $\operatorname{Out}\left(F_{n}\right)$ on free factorizations.

There are a few properties known about these spaces and their siblings. Hatcher showed that the sphere complex, which contains Outer Space as a dense subspace, is contractible (this gives an alternate proof of contractibility of Outer Space [9], as the contraction restricts to a contraction of Outer Space). Hatcher and Vogtmann showed that the edge splitting and free factor complexes - at least the $\operatorname{Aut}\left(F_{n}\right)$ versions of them, where we do not identify objects which differ by conjugation - are both $(n-2)$-spherical [16], [17] (again, Hatcher and Vogtmann use the terminology 'free factorization complex' in place of 'edge splitting complex'). It seems to be an open question whether the $\operatorname{Out}\left(F_{n}\right)$ versions of these complexes are also spherical. To study $\operatorname{Out}\left(F_{n}\right)$, Guirardel [13] has introduced a notion of intersection form for actions of a group $G$ on metric simplicial trees (that is, $G$-trees), which can be applied to 
the specific case of $G=F_{n}$. Behrstock, Bestvina, and Clay [2] used Guirardel's intersection form to describe the effect of applying fully irreducible automorphisms to vertices in $\mathscr{E} S_{n}^{1}$. They also discuss the edge splitting complex (therein called the splitting complex though HNN extensions are not allowed, as in [20]), and a related complex called the subgraph complex. Kapovich and Lustig [19], [22] have also introduced an intersection form (distinct from Guirardel's), inspired by the work of Bonahon [8]. Kapovich and Lustig have shown that $\mathcal{E} S_{n}^{1}$ and $\mathcal{F F}_{\mathcal{F}_{n}}{ }_{n}$, as well as their intersection graph and 6 other related graphs, all have infinite diameter [20]. Recently, Yakov Berchenko-Kogan [4] characterized vertices of distance 2 apart in the ellipticity graph, a graph quasiisometric to $\mathcal{F F}_{\mathcal{F}}{ }_{n}^{1}$, using Stallings foldings. This effectively characterizes adjacent vertices in $\mathcal{F F}_{\mathcal{F}}{ }_{n}$ (the latest version of [4] states that the equivalent results were obtained earlier using different language in [28] and [21]). Further, Day and Putman [10] proved that another curve complex analogue, the complex of partial bases, is connected and simply connected. The 1-skeleton of this complex is called the primitivity graph in [20], where it is also claimed that this graph is quasiisometric to the free factorization graph $\mathscr{F}_{\mathcal{F}}{ }_{n}^{1}$. Aramayona and Souto have shown that $\operatorname{Out}\left(F_{n}\right)$ is precisely the group of simplicial automorphisms of the free splitting complex $\mathcal{F} S_{n}[1]$.

The study of the coarse geometry of $\operatorname{Out}\left(F_{n}\right)$ is currently moving very quickly. Since the time that this paper first came out, great progress has been made in this area. In particular, recent works of Bestvina and Feighn [7] and Handel and Mosher [14] have shown that the free factor graph and the free splitting graph, respectively, are hyperbolic spaces on which $\operatorname{Out}\left(F_{n}\right)$ acts. Before these results were known, Behrstock, Bestvina, and Clay [2] suggested that "there is a hope that a proof of hyperbolicity of the curve complex generalizes to the [edge splitting] complex". However, we intend to prove:

Theorem 5.4. For $n>2$, the space $\mathcal{E} S_{n}^{1}$ (and hence $\mathcal{E} S_{n}$ ) contains a quasiisometrically embedded copy of $\mathbb{R}^{m}$ for every $m \geq 1$.

Our proof relies on attaining an understanding of distances in $\varepsilon S_{n}^{1}$. To do so, we associate vertices of $\varepsilon S_{n}^{1}$ with bases of $F_{n}$. With this association, we are able to completely characterize (up to distance 4) the length of a path in $\& S_{n}^{1}$ via a simple algebraic notion which we call number of index changes. This characterization is made precise in Theorem 3.2 and the preceding discussion.

To utilize this translation from geometry to algebra, we then introduce an algebraic notion of complexity of a basis, which we call $i$-length. The notion of $i$-length is itself based roughly on having many subwords of elements of the basis with complicated Whitehead graphs. Our techniques, in turn, use a theorem of Stallings (see Section 4 for details). The bulk of this paper aims to translate this $i$-length notion of how complicated a basis is into a lower bound on distances between vertices in $\mathcal{E} S_{n}^{1}$, as shown in the following theorem:

Theorem 5.3. Let $\boldsymbol{x}$ be a basis of $F_{n}$, expressed in terms of a fixed standard basis $\boldsymbol{a}$. 
The distance between a vertex of $\mathcal{E} S_{n}^{1}$ associated to $\boldsymbol{a}$ and one associated to $\boldsymbol{x}$ is at least $\frac{|\boldsymbol{x}|_{i}}{24}-1$, where $|\boldsymbol{x}|_{i}$ is the $i$-length of $\boldsymbol{x}$.

As immediate corollaries of Theorem 5.4, we obtain:

Corollary 5.5. The space $\mathcal{E} \mathcal{S}_{n}^{1}$ is not Gromov hyperbolic.

In other words, $\mathcal{E} S_{n}^{1}$ is not the 'correct' curve complex analogue for $\operatorname{Out}\left(F_{n}\right)$. This shows that the 'hope' of [2] is a false one, at least for the edge splitting graph. Indeed, it might be expected that the edge splitting graph is not hyperbolic: edge splittings correspond to separating spheres in the sphere complex. But in the mapping class group world, the subcomplex of the curve complex induced by only allowing separating curves is itself not hyperbolic [27].

Corollary 5.6. The space $\& S_{n}^{1}$ has infinite asymptotic dimension. The dimension of every asymptotic cone of $\mathcal{E} S_{n}^{1}$ is infinite.

To the authors' knowledge, this is the only naturally defined space which has infinite asymptotic dimension and a natural cocompact group action of a group which is not known to have infinite asymptotic dimension. Thompson's group $F$ acts on a cube complex with arbitrary-dimensional quasiflats [12], but has infinite asymptotic dimension (moreover, it is proved in [11] that $F$ has exponential dimension growth). Via private communication, Moon Duchin claims that the Cayley graph of $\mathbb{Z}$ with respect to the infinite generating set consisting of powers of 2 has arbitrary-rank quasiflats. Thus, we have a group with finite asymptotic dimension acting on a space with infinite asymptotic dimension. However, this action is not cocompact: the quotient is a graph with one vertex and infinitely many edges. Both the mapping class group [5] and arithmetic groups [18] have finite asymptotic dimension, so the analogy between $\operatorname{Out}\left(F_{n}\right)$ and these groups suggests that $\operatorname{Out}\left(F_{n}\right)$ may in fact have finite asymptotic dimension.

There is a further interesting consequence of Theorem 5.4. There is a natural map $\mathrm{id}^{*}$ from $\mathcal{E} S_{n}^{1}$ to $\mathcal{F F}_{\mathcal{F}}{ }_{n}^{1}$ induced by the identity map on the vertex set. This map id* is 1-Lipshitz: if two free factorizations have a common refinement, then any nontrivial elliptic element of the common refinement will have translation length 0 on both of the corresponding Bass-Serre trees. The quasiflats described in the proof of Theorem 5.4 are in fact such that, for every quasiflat, there exists a common elliptic element such that every vertex in that quasiflat has a representative where one factor contains the common elliptic element. Thus,

Corollary 5.7. The map $\mathrm{id}^{*}: \mathcal{E} S_{n}^{1} \rightarrow \mathcal{F F}^{\mathcal{F}}{ }_{n}^{1}$ is not a quasiisometry. Moreover, there is no coarsely $\operatorname{Out}\left(F_{n}\right)$-equivariant quasiisometry between $\& S_{n}^{1}$ and $\mathcal{F}_{\mathcal{F}}{ }_{n}^{1}$.

An analogous results hold true for the relationships between the free factorization graph $\mathcal{E} S_{n}^{1}$ and the free factor graph $\mathscr{F}_{n}^{1}$ and between $\mathcal{E} S_{n}^{1}$ and the free splitting graph $\mathcal{F} S_{n}^{1}$. There is a natural (coarsely well defined for $n>2$ ) map $\Sigma: \mathcal{E} S_{n}^{1} \rightarrow \mathscr{F}_{n}^{1}$ 
defined by sending a vertex $[A * B]$ in $\mathcal{E} S_{n}^{1}$ to the vertex $[A]$ in $\mathcal{F}_{n}{ }_{n}$. Also there is a natural embedding $\imath: \mathcal{E} S_{n}^{1} \rightarrow \mathcal{F}_{n}^{1}$ defined by sending a vertex $[A * B]$ in $\mathscr{E} S_{n}^{1}$ to the vertex $[A * B]$ in $\mathscr{F} S_{n}^{1}$, which is quasisurjection. However, neither of the above maps is a quasiisometry:

Corollary 5.8. The maps $\Sigma: \mathcal{E} S_{n}^{1} \rightarrow \mathcal{F}_{n}^{1}$ and $\imath: \mathcal{E} S_{n}^{1} \rightarrow \mathcal{F}_{n}^{1}$ are not quasiisometries. Moreover, there is no coarsely $\operatorname{Out}\left(F_{n}\right)$-equivariant quasiisometry between $\mathcal{E} S_{n}^{1}$ and $\mathcal{F}_{n}^{1}$, and between $\mathcal{E} S_{n}^{1}$ and $\mathcal{F}_{S_{n}}^{1}$.

The last corollary provides a negative answer to a question of Bestvina and Feighn (the first half of Question 4.4 in [6]).

Finally, taking into account the recent results of Bestvina and Feighn [7] and of Handel and Mosher [14] claiming hyperbolicity of $\mathscr{F}_{n}^{1}$ and $\mathscr{F} S_{n}^{1}$ respectively, one immediately obtains a more general corollary:

Corollary 5.9. The edge splitting graph $\mathcal{E} \delta_{n}^{1}$ is not quasiisometric to any of $\mathcal{F F}{ }_{n}^{1}$, $\mathcal{F}_{n}^{1}$ and $\mathcal{F} \mathcal{S}_{n}^{1}$.

This paper is organized as follows. We begin in Section 2 by describing three ways of viewing an element of $\operatorname{Aut}\left(F_{n}\right)$. Being able to translate between these three perspectives will be useful at various points in the later proofs. In Section 3, we describe how to view vertices in $\mathcal{E} S_{n}^{1}$ and $\mathcal{F F}_{F}{ }_{n}^{1}$ as pairs consisting of an element of $\operatorname{Aut}\left(F_{n}\right)$ and a proper nonempty subset of $\{1,2, \ldots, n\}$ up to certain identifications. This viewpoint allows us to interpret distances in $\mathcal{E} S_{n}^{1}$ algebraically, in terms of elements of $\operatorname{Aut}\left(F_{n}\right)$, culminating in Theorem 3.2.

Most of the details in the paper are in Section 4. There we introduce the notion of $i$-length. For technical reasons, we use three different notions of $i$-length: fixing some basis $\boldsymbol{a}$ of $F_{n}$, we have simple $i$-length for abstract words over $\boldsymbol{a}$, conjugate reduced $i$-length for subwords written over $\boldsymbol{a}$ of some other basis of $F_{n}$, and full $i$-length for bases of $F_{n}$ themselves. In Section 4, we describe properties of each of these notions of $i$-length in turn. The section builds up to, and ends with, Theorem 5.3.

Finally in Section 5, we relate the algebraic notion of $i$-length to distances in $\& S_{n}^{1}$, and use this relationship to prove Theorem 5.4 and its corollaries, described above.

The authors would like to thank Ilya Kapovich, Diane Vavrichek, Keith Jones, Dan Farley, and Karen Vogtmann for useful conversations on this material, and Mladen Bestvina, Matt Clay, Michael Handel, and especially Lee Mosher for useful comments. Finally, we appreciate the comments and suggestions of anonymous referees that have helped to enhance the paper.

\section{Three interpretations of $\operatorname{Aut}\left(F_{n}\right)$}

Fix a basis $\boldsymbol{a}=\left(a_{1}, \ldots, a_{n}\right)$ of $F_{n}$, considered as an ordered tuple. The group of all automorphisms of $F_{n}$ has many interpretations. For our purposes, we will use three of these interpretations, as follows. 
The first interpretation of $\operatorname{Aut}\left(F_{n}\right)$ is as in bijective correspondence with the set of ordered bases of $F_{n}$. Consider a basis $\boldsymbol{x}=\left(x_{1}, \ldots, x_{n}\right)$ of $F_{n}$ as an ordered tuple. As $\boldsymbol{x}$ is a basis, there exists an automorphism $\phi_{\boldsymbol{x}}$ which maps $\boldsymbol{a}$ to $\boldsymbol{x}$; as automorphisms are uniquely specified by their action on a given generating set, $\phi_{\boldsymbol{x}}$ is unique. Thus, $\operatorname{Aut}\left(F_{n}\right)$ as a set is in bijective correspondence with the set

$$
\boldsymbol{X}:=\left\{\boldsymbol{x}=\left(x_{1}, \ldots, x_{n}\right) \in F_{n}^{n} \mid \boldsymbol{x} \text { is an ordered basis }\right\} .
$$

The second interpretation of $\operatorname{Aut}\left(F_{n}\right)$ is as products of elementary Nielsen automorphisms. Nielsen [26] described a generating set for $\operatorname{Aut}\left(F_{n}\right)$ consisting of four types of generators:

Definition 2.1. An elementary Nielsen automorphism is an automorphism of $F_{n}$ for which there exist indices $i, j$ such that $i \neq j, a_{k} \mapsto a_{k}$ for $k \neq i, j$, and one of the following four possibilities holds:

$$
\begin{aligned}
s_{i j}: & a_{i} \leftrightarrow a_{j} \\
t_{i}: & a_{i} \mapsto a_{i}^{-1}, \\
a_{i j}: & a_{i} \mapsto a_{i} a_{j} \\
a_{i j}^{-1}: & a_{i} \mapsto a_{i} a_{j}^{-1}
\end{aligned}
$$

The group operation in $\operatorname{Aut}\left(F_{n}\right)$ with respect to Nielsen automorphisms is function composition, where automorphisms are composed as functions, right-to-left. Note a Nielsen automorphism $\phi$ acts on the Cayley graph of $\operatorname{Aut}\left(F_{n}\right)$ via the usual left action. We can interpret this action on the vertices of the Cayley graph in terms of the correspondence between $\operatorname{Aut}\left(F_{n}\right)$ and $X$ : an automorphism $\phi$ acting on a basis $\boldsymbol{x} \in \boldsymbol{X}$ has image $\phi(\boldsymbol{x})=\left(\phi x_{1}, \ldots, \phi x_{n}\right)=\phi \circ \phi_{\boldsymbol{x}}(\boldsymbol{a})$.

The third interpretation of $\operatorname{Aut}\left(F_{n}\right)$ is as the group of Nielsen transformations. A Nielsen transformation is an action on the set of ordered bases of $F_{n}$ (that is, on $\operatorname{Aut}\left(F_{n}\right)$, by the first interpretation) which may be decomposed as a product of elementary Nielsen transformations. These elementary Nielsen transformations are free-group analogues of the elementary row operations in $G L_{n}(\mathbb{Z})=\operatorname{Aut}\left(\mathbb{Z}^{n}\right)$, and, in fact, induce the elementary row operations under the abelianization map $F_{n} \rightarrow \mathbb{Z}^{n}$. There are four kinds of elementary Nielsen transformations:

Definition 2.2. An elementary Nielsen transformation is a map on the set of ordered bases $\boldsymbol{X}=\left\{\boldsymbol{x}=\left(x_{1}, \ldots, x_{n}\right)\right\}$ of $F_{n}$ for which there exist indices $i, j$ such that $i \neq j, x_{k} \mapsto x_{k}$ for $k \neq i, j$, and one of the following four possibilities hold:

(1) $\sigma_{i j}: x_{i} \leftrightarrow x_{j}$,

(2) $\tau_{i}: x_{i} \mapsto x_{i}^{-1}$,

(3) $\alpha_{i j}: x_{i} \mapsto x_{i} x_{j}$,

(4) $\alpha_{i j}^{-1}: x_{i} \mapsto x_{i} x_{j}^{-1}$.

Elementary Nielsen transformations of type (3) and (4) are called transvections. 
The group operation in $\operatorname{Aut}\left(F_{n}\right)$ with respect to Nielsen transformations is again composition, but transformations are composed left-to-right. Nielsen transformations act on $\boldsymbol{X}$ on the right.

The isomorphism between the groups generated by Nielsen automorphisms and by Nielsen transformations is clear: the isomorphism is $s_{i j} \mapsto \sigma_{i j}, t_{i} \mapsto \tau_{i}, a_{i j} \mapsto \alpha_{i j}$. Thus, a word in elementary Nielsen transformations may be considered as a word in Nielsen automorphisms, written in the same order, but with the order of composition reversed and the action on $\boldsymbol{X}$ on the left instead of the right.

These three interpretations are different aspects of the same concept: the set $\boldsymbol{X}$ may be viewed as the vertices of the Cayley graph of $\operatorname{Aut}\left(F_{n}\right)$; elementary Nielsen automorphisms form a generating set of $\operatorname{Aut}\left(F_{n}\right)$ and their action on $\boldsymbol{X}$ corresponds to the left action of this generating set on its Cayley graph. This is the action such that an automorphism $g$ takes a vertex $v$ to $g v$, and takes an edge connecting $v$ to $v a$ to an edge connecting $g v$ and $g v a$ for each generator $a$ of $\operatorname{Aut}\left(F_{n}\right)$. Elementary Nielsen transformations form the same generating set, but with the action on $\boldsymbol{X}$ being an interpretation of the right action of the generating set on the vertices of its Cayley graph. When restricted to the action of a generator $a$ of $\operatorname{Aut}\left(F_{n}\right)$, it simply moves a vertex $v$ across the edge connecting $v$ to $v a$ to the vertex $v a$. However, this right action does not extend to the edges of the Cayley graph.

In his seminal paper [26], Nielsen presented a method for transforming a finite generating set for a subgroup of a free group into a free basis for that subgroup using elementary Nielsen transformations. Nielsen's method is essentially a finite reduction process, at every step of which a Nielsen transformation is used to 'simplify' the finite generating set. In Lemma 4.20 we will apply this process to the bases of $F_{n}$ and will use the following fact, whose proof follows from the proof of Theorem 3.1 in [24].

Proposition 2.3. For every basis $\boldsymbol{x}$ of a free group $F_{n}$ there is a sequence of elementary Nielsen transformations $\left(\delta_{j}\right), 1 \leq j \leq t$ taking the standard basis a of $F_{n}$ to $\boldsymbol{x}=\boldsymbol{a} \delta_{1} \ldots \delta_{t}$ such that the sum of the lengths (with respect to $\boldsymbol{a}$ ) of elements in the intermediate bases $\boldsymbol{a} \delta_{1} \ldots \delta_{j}$ is a nondecreasing sequence.

\section{Vertices and edges in $\varepsilon S_{n}^{1}$}

We wish to view the spaces $\mathcal{E} S_{n}^{1}$ and $\mathcal{F}_{\mathcal{F}}{ }_{n}^{1}$ on which $\operatorname{Out}\left(F_{n}\right)$ acts in the language of ordered tuples, so that we may apply the dictionary of Section 2 equating tuples, Nielsen automorphisms, and Nielsen transformations.

We begin with an observation on elements of $\operatorname{Out}\left(F_{n}\right)$. An element of $\operatorname{Out}\left(F_{n}\right)$ is a coset of $\operatorname{Aut}\left(F_{n}\right)$ with respect to the subgroup $\operatorname{Inn}\left(F_{n}\right)$. As such, an element of $\operatorname{Out}\left(F_{n}\right)$ may be represented by many different $n$-tuples. In general, we think of an element of $\operatorname{Out}\left(F_{n}\right)$ as a tuple up to conjugation.

Now consider the graphs $\mathcal{E} \delta_{n}^{1}$ and $\mathcal{F}_{\mathcal{F}}{ }_{n}^{1}$. These graphs have the same vertex 
set: vertices correspond to free factorizations of $F_{n}$ into two nontrivial factors up to conjugation. We wish to interpret an arbitrary free factorization of $F_{n}$ into two factors as a tuple, together with an index set, up to certain equivalences. Let $\delta$ denote the set of all proper nonempty subsets of $\{1, \ldots, n\}$. We will call an element of $\delta$ an index set. Then a tuple $\boldsymbol{x}=\left(x_{1}, \ldots, x_{n}\right)$ together with some index set $S \in \mathcal{S}$ yields a free factorization of $F_{n}$ as $\left\langle\boldsymbol{x}_{S}\right\rangle *\left\langle\boldsymbol{x}_{\bar{S}}\right\rangle$, where $\boldsymbol{x}_{S}:=\left\{a_{i} \in \boldsymbol{x} \mid i \in S\right\}$ and $\bar{S}:=\{1, \ldots, n\}-S$. Every free factorization may be represented as a tuple/index set pair, but a given free factorization may be represented by multiple tuple/index set pairs: any tuple/index set pairs which differ by a self-map of $\operatorname{Aut}\left(F_{n}\right) \times \delta$ preserving the associated free factorization up to conjugation should be identified.

Every such map can be written as a composition of four types of self-maps, defined by their action on $(\boldsymbol{x}, S) \in \operatorname{Aut}\left(F_{n}\right) \times S$ as follows:

(1) conjugation of $\boldsymbol{x}$ without changing $S$,

(2) permutation of $\{1, \ldots, n\}$ applied to both $\boldsymbol{x}$ and $S$,

(3) exchanging $S$ for $\bar{S}$ and leaving $\boldsymbol{x}$ unchanged,

(4) applying transformation $\phi$ of $\operatorname{Aut}\left(F_{n}\right)$ fixing the free factors in the factorization setwise (i.e. $\left\langle\boldsymbol{x}_{S}\right\rangle=\left\langle(\boldsymbol{x} \phi)_{S}\right\rangle$ and $\left\langle\boldsymbol{x}_{\bar{S}}\right\rangle=\left\langle(\boldsymbol{x} \phi)_{\bar{S}}\right\rangle$ ) without changing $S$.

The transformation $\phi$ in the last item is called an $S$-transformation. If there exists $S \in S$ such that $\phi$ is an $S$-transformation, we call $\phi$ an $\delta$-transformation.

Note that any self-map from the group mentioned above may be realized as composition of the form $m_{1} m_{2} m_{3} m_{4}$, where $m_{i}$ is a self-map of type (i).

With this interpretation of vertices of $\& \delta_{n}^{1}$, consider edges of $\& S_{n}^{1}$. Two vertices represented by $A_{1} * B_{1}$ and $A_{2} * B_{2}$ of $\& S_{n}^{1}$ are adjacent if there exists a common refinement of conjugates of the free factorizations. Such a common refinement is of the form $A * C * B$, where for some elements $g$ and $h$ of $F_{n}$ we have $A_{1}^{g}=A * C$, $B_{1}^{g}=B, A_{2}^{h}=A$, and $B_{2}^{h}=C * B$. Without loss of generality we can assume that $h$ is trivial. If $(\boldsymbol{x}, S)$ is the vertex corresponding to the free factorization $A_{1} * B_{1}$, then $\left(x^{g}, S\right)$ represents the same vertex of $\mathcal{E} S_{n}^{1}$ and the refinement $A_{1}^{g}=A * C$ corresponds to applying to $\boldsymbol{x}^{g}$ a transformation $\phi$ of $F_{n}$ taking $\boldsymbol{x}_{S}^{g}$ to a basis for $A$ union a basis for $C$ and preserving $B_{1}^{g}$. Note $\phi$ fixes both $\left\langle x_{S}^{g}\right\rangle$ and $\left\langle x_{\bar{S}}^{g}\right\rangle$, and so is an $S$-transformation. Then changing $(A * C) * B$ to $A *(C * B)$ simply corresponds to subtracting from $S$ the indices of elements in $\phi\left(x^{g}\right)$ corresponding to a basis for $C$. Of course, by exchanging $S$ for $\bar{S}$, we could have instead added elements to $S$, which corresponds to subtracting elements from $\bar{S}$. Thus, changing $(A * C) * B$ to $A *(C * B)$ corresponds to replacing $S$ with a proper subset of either $S$ or $\bar{S}$. We call index sets $S$ and $S^{\prime}$ from $S$ compatible if either $S^{\prime}$ or $\overline{S^{\prime}}$ is a proper subset of either $S$ or $\bar{S}$.

Thus, up to conjugation, all edges from the vertex corresponding to $(\boldsymbol{x}, S)$ are precisely characterized by a transformation fixing $\left\langle\boldsymbol{x}_{S}\right\rangle$ and $\left\langle\boldsymbol{x}_{\bar{S}}\right\rangle$, followed by replacing $S$ with a compatible element of $\delta$. We have shown: 
Lemma 3.1. The set of edges in $\mathcal{E} S_{n}^{1}$ from a vertex $(\boldsymbol{x}, S)$ is determined by: a conjugation of $\boldsymbol{x}$, an $S$-transformation, and a choice of new index set compatible with $S$.

An edge path $p$ from the vertex represented by $\left(\boldsymbol{x}, S_{0}\right)$ in $\mathcal{E} \delta_{n}^{1}$ is described by a sequence: a conjugation $\gamma_{0}$ of $\boldsymbol{x}$, an $S_{0}$-transformation $\phi_{0}$, a change of index set to $S_{1}$, a conjugation $\gamma_{1}$ of $\boldsymbol{x}^{\gamma_{0}} \phi_{0}$, an $S_{1}$-transformation $\phi_{1}$, a change of index set to $S_{2}$, etc.

On such an edge path $p$, for any $i$, let $\left(x(i), S_{i}\right)$ be a representative of the vertex on the edge path immediately before $\phi_{i}$ is to be applied. Then by construction we have

$$
\begin{aligned}
\boldsymbol{x}(i) & =\left(\ldots\left(\left(\boldsymbol{x}^{\gamma_{0}} \phi_{0}\right)^{\gamma_{1}} \phi_{1}\right)^{\gamma_{2}} \ldots\right)^{\gamma_{i-1}} \phi_{i-1} \\
& =\left(\boldsymbol{x} \phi_{0} \phi_{1} \ldots \phi_{i-1}\right)\left(\ldots\left(\left(\gamma_{0}\right) \phi_{0} \gamma_{1}\right) \phi_{1} \gamma_{2} \ldots\right) \phi_{i-1} .
\end{aligned}
$$

As vertices of $\mathcal{E} S_{n}^{1}$ are only defined up to conjugation, we may assume without loss of generality that all of the conjugators $\gamma_{i}$ are trivial and

$$
\boldsymbol{x}(i)=\boldsymbol{x} \phi_{0} \phi_{1} \ldots \phi_{i-1} .
$$

The set $S_{i}$ is not determined uniquely by $\phi_{i}$, as $\phi_{i}$ may be an $S$-transformation for many index sets $S$. However, for any such $S$, the vertex $(\boldsymbol{x}(i), S)$ is of distance at most 2 away from each of the vertices $\left(x(i-1), S_{i-1}\right),\left(x(i), S_{i}\right)$, and $\left(x(i+1), S_{i+1}\right)$ in $\varepsilon S_{n}^{1}$, as follows. That $(\boldsymbol{x}(i), S)$ is distance at most 2 from $\left(\boldsymbol{x}(i-1), S_{i-1}\right)$ follows from applying $\phi_{i-1}$ to $\left(\boldsymbol{x}(i-1), S_{i-1}\right)$ and then changing the index set to $S$, which requires one edge in $\mathcal{E} S_{n}^{1}$ if $S_{i-1}$ and $S$ are compatible and 2 edges otherwise. That $(\boldsymbol{x}, S)$ is distance at most 2 from $\left(\boldsymbol{x}(i), S_{i}\right)$ follows from applying the identity transformation to $\left(x(i), S_{i}\right)$ (note the identity transformation is indeed an $S_{i}$-transformation) and then changing index set to $S_{i}$. Finally, for the vertex $\left(\boldsymbol{x}(i+1), S_{i+1}\right)$, since $\phi_{i}$ is an $S$-transformation, $\left(\boldsymbol{x}(i+1), S_{i+1}\right)$ is the vertex obtained from $(\boldsymbol{x}, S)$ by applying $\phi_{i}$ to $\boldsymbol{x}(i)$ and then changing the index set to $S_{i+1}$.

Thus, up to distance 2 at every vertex on the path $p$, the path $p$ is determined by the sequence of transformations $\phi_{0}, \phi_{1}, \ldots, \phi_{k}$ (see Figure 1). Note that we may reverse this procedure: take a sequence of transformations $\phi_{0}, \phi_{1}, \ldots, \phi_{k}$ such that each $\phi_{i}$ is an $\mathcal{S}$-transformation, choose any $S_{i}^{\prime} \in \mathcal{S}$ such that $\phi_{i}$ is an $S_{i}^{\prime}$-transformation, and obtain an edge path in $\mathscr{E} S_{n}^{1}$, which is uniquely defined up to distance 2 at each vertex.

A geodesic in $\mathcal{E} S_{n}^{1}$ is then easy to describe. A geodesic, up to distance 2 at each vertex, is an edge path $\phi_{0}, \phi_{1}, \ldots, \phi_{k}$ such that the transformation $\phi=\phi_{0} \phi_{1} \ldots \phi_{k}$ is not a product of fewer than $k+1 \delta$-transformations with the property that the neighboring transformations are $\delta$-transformations with respect to compatible index sets.

For a given word $w$ in the generating set for $\operatorname{Aut}\left(F_{n}\right)$ consisting of elementary Nielsen transformations and the identity transformation, we say that $w$ has at most $k$ 


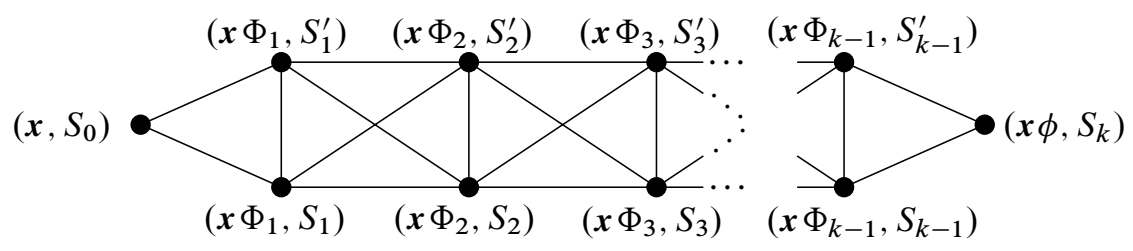

Figure 1. Shown are two edge paths from the vertex $\left(x, S_{0}\right)$ to the vertex $\left(x \phi, S_{k}\right)$ in $\varepsilon \delta_{n}^{1}$ using the notation of this section. Here, we let $\Phi_{i}:=\phi_{0} \ldots \phi_{i-1}$ denote the composition of $\mathcal{S}$-transformations, where $\phi_{i}$ is an $S_{i-1}$-transformation. Thus, $\boldsymbol{x}(i)=\boldsymbol{x} \Phi_{i}=\boldsymbol{x} \phi_{0} \ldots \phi_{i-1}$. The lower path represents the edge path described in the text, and is represented by the sequence of transformations $\phi_{0}, \ldots, \phi_{k}$. The upper path represents an edge path reconstructed from the $\mathcal{S}$-transformations $\phi_{0}, \ldots, \phi_{k}$ by, for each $i=1, \ldots, k-1$, choosing an arbitrary index set $S_{i}^{\prime}$ compatible with $S_{i-1}^{\prime}$ such that $\phi_{i}$ is an $S_{i}^{\prime}$-transformation. Horizontal edges in the figure are edges in $\& S_{n}^{1}$, and vertical and diagonal edges mean that the distance between two vertices in $\mathcal{E} S_{n}^{1}$ is at most 2.

index changes if $w$ may be expressed as a product of $k+1$ disjoint subwords, each of which is an $\delta$-transformation and the neighboring subwords are $\delta$-transformations with respect to compatible index sets. If $k$ is minimal over all such products, we say $w$ requires $k$ index changes. Since the product of $S$-transformations is an $S$ transformation, we can rephrase the preceding paragraph in the form of the following theorem.

Theorem 3.2. A geodesic in $\mathcal{E} S_{n}^{1}$ is represented by a product of $\delta$-transformations with the minimal number of index changes, such that the neighboring $S$-transformations correspond to compatible index sets. Moreover, a geodesic of length $k$ in $\& S_{n}^{1}$ requires between $k-4$ and $k$ compatible index changes.

We will use this characterization to describe lower bounds on distances in $\& S_{n}^{1}$ based on properties of the associated transformations in Sections 4 and 5.

We end this section by noting that there is a similar characterization of roses in the spine $K_{n}$ of outer space as tuples, up to conjugation and signed permutation (the signed permutations correspond to graph isomorphisms). With this interpretation, there are canonical Lipschitz maps from $K_{n}$ to $\mathcal{E} \delta_{n}^{1}$ to $\mathcal{F}_{\mathcal{F}}{ }_{n}^{1}$. It is also worth noting that the quasiisometry between $\operatorname{Out}\left(F_{n}\right)$ and $K_{n}$ may be stated in this language: Let $K_{n}^{\prime}$ be the graph whose vertices are the marked roses of $K_{n}$ and whose edges correspond to marked roses lying on a common 2-cell in $K_{n}$. Then $K_{n}$ is 2-biLipschitz equivalent to the graph $K_{n}^{\prime}$, and $K_{n}^{\prime}$ is biLipschitz equivalent to the Cayley graph of $\operatorname{Out}\left(F_{n}\right)$ with respect to the generating set of elementary Whitehead transformations: $K_{n}^{\prime}$ is the Schreier graph of $\operatorname{Out}\left(F_{n}\right)$ with respect to this generating set and the finite subgroup of signed permutations. 


\section{The notion of $i$-length}

In this section, we define the notion of $i$-length and analyze its properties. This notion is an algebraic tool that will be used to estimate distances in $\varepsilon S_{n}^{1}$. We use the concept of $i$-length to refer to a measure of complexity of 3 different kinds of objects: abstract words in the generators of $F_{n}$, subwords of bases of $F_{n}$, and bases of $F_{n}$ themselves. Our 3 concepts of $i$-length are: simple $i$-length, conjugate reduced $i$-length, and full $i$-length, respectively. We use simple $i$-length to define conjugate reduced $i$-length, and conjugate reduced $i$-length to define full $i$-length. After defining the three notions of $i$-length, we will analyze the properties of each in turn.

Throughout this section, we fix a standard basis $\boldsymbol{a}=\left\{a_{1}, \ldots, a_{n}\right\}$ of $F_{n}$ once and for all.

4.1. Defining $i$-length. We motivate our definition of $i$-length with an example.

Let $H:=\left\langle a_{1}, \ldots, a_{n-1}\right\rangle$ denote the subgroup of $F_{n}$ of rank $n-1$ corresponding to ignoring the generator $a_{n}$. Consider the vertex $v_{0}:=\left[H *\left\langle a_{n}\right\rangle\right]$ as a basepoint in $\mathcal{E} S_{n}^{1}$, and think about moving in $\mathcal{E} S_{n}^{1}$ to the vertex $v=\left[H *\left\langle a_{n} h\right\rangle\right]$, where $h$ is an arbitrary element of $H$. Let $d$ denote the distance between $v_{0}$ and $v$ in $\& S_{n}^{1}$.

If $h$ is nontrivial, then $v \neq v_{0}$, as there is clearly no way of using conjugation to remove occurrences of all elements of $H$ from the second factor of any representative of $v$. Moreover, as $v_{0}$ and $v$ both have the same index set, by Theorem 3.2, when $h$ is nontrivial we have $d \geq 2$.

If $h$ is a primitive element in $H$, then $d=2$, as follows. Let $h_{2}, \ldots, h_{n-1}$ denote elements of $H$ such that $\left\{h, h_{2}, \ldots, h_{n-1}\right\}$ forms a basis for $H$. Then

$$
\left\langle h, h_{2}, \ldots, h_{n-1}\right\rangle *\left\langle a_{n}\right\rangle
$$

is a representative of $v_{0}$, and

$$
\left\langle h, h_{2}, \ldots, h_{n-1}\right\rangle *\left\langle a_{n} h\right\rangle
$$

is a representative of $v$. Thus, $\left[\left\langle h_{2}, \ldots, h_{n-1}\right\rangle *\left\langle h, a_{n}\right\rangle\right]$ is a vertex which is adjacent to both $v$ and $v_{0}$.

If $h$ is a power of a primitive element in $H$, the same argument again shows that $d=2$. Figure 2 shows the path of length 2 connecting $\left[H *\left\langle a_{n}\right\rangle\right]$ and $\left[H *\left\langle a_{n} h\right\rangle\right]$ for

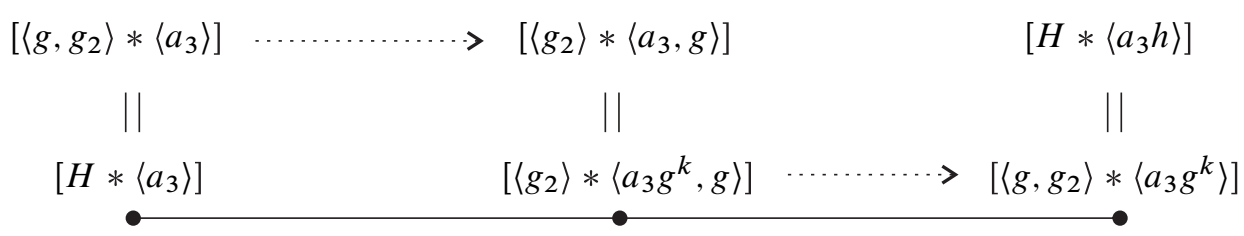

Figure 2. A path of length 2 in $\varepsilon s_{3}^{1}$. 
$n=3, h=g^{k}$, where $g$ is primitive in $H$ and $g_{2}$ is some coprimitive with $g$ element such that $\left\langle g, g_{2}\right\rangle=H$. Repeating the above argument shows that, if we know that $h$ is a product of $j$ powers of primitive elements in $H$, then $d \leq 2 j$. To obtain a lower bound on $d$, we need to at least minimize $j$. Thus, we need to consider how to detect how many powers of primitives are needed to form $h$.

One property of a (power of a) primitive element $h$ of $H$ is a classical result of Whitehead, which states that the Whitehead graph of $h$, considered as a reduced word in the alphabet $\left(\boldsymbol{a}-\left\{a_{n}\right\}\right)^{ \pm 1}$, must have a cut vertex, defined as follows.

Definition 4.1 (Whitehead graph). For a set of freely reduced words $\boldsymbol{x}=\left\{x_{1}, \ldots, x_{k}\right\}$ in the alphabet $\boldsymbol{a} \cup \boldsymbol{a}^{-1}$, define the Whitehead graph $\Gamma_{\boldsymbol{a}}(\boldsymbol{x})$ as follows. The set of vertices of $\Gamma_{\boldsymbol{a}}(\boldsymbol{x})$ is identified with the set $\boldsymbol{a} \cup \boldsymbol{a}^{-1}$. For every $x_{i} \in \boldsymbol{x}$ of length $n, x_{i}$ contributes exactly $n-1$ edges to $\Gamma_{\boldsymbol{a}}(\boldsymbol{x})$, one for each pair of consecutive letters in $x_{i}$. The edge added for a $a_{i} a_{j}$ is from the vertex $a_{i}$ to the vertex $a_{j}^{-1}$. The augmented Whitehead graph $\widehat{\Gamma}_{\boldsymbol{a}}(\boldsymbol{x})$ is the Whitehead graph $\Gamma_{\boldsymbol{a}}(\boldsymbol{x})$ together with an additional edge for each $x_{i} \in \boldsymbol{x}$, from the last letter of $x_{i}$ to the inverse of the first letter. In particular, a word $x_{i}=a_{j}$ of length 1 contributes exactly one edge, from $a_{j}$ to $a_{j}^{-1}$, to $\widehat{\Gamma}_{\boldsymbol{a}}(\boldsymbol{x})$. For a single word $w$, we abuse notation and write $\Gamma_{\boldsymbol{a}}(w)$ for $\Gamma_{\boldsymbol{a}}(\{w\})$ and $\widehat{\Gamma}_{\boldsymbol{a}}(w)$ for $\widehat{\Gamma}_{\boldsymbol{a}}(\{w\})$.

If $\boldsymbol{x}$ is cyclically reduced and is a basis for $\langle\boldsymbol{x}\rangle \subset F_{n}$ then the augmented Whitehead graph of a set of freely reduced words $\boldsymbol{x}$ is graph-isomorphic to the link of the unique vertex in the presentation 2-complex of the group $F_{n} /\langle\langle\boldsymbol{x}\rangle\rangle$ generated by $a_{1}, \ldots, a_{n}$ with relations $x_{1}, \ldots, x_{k}$.

Note that a Whitehead graph (or augmented Whitehead graph) may have multiple edges. Loops at a vertex may appear only in an augmented Whitehead graph and if and only if at least one of the words in $\boldsymbol{x}$ is not cyclically reduced. An example of the augmented Whitehead graph, namely $\widehat{\Gamma}_{\left\{a_{1}, a_{2}, a_{3}, a_{4}\right\}}\left(a_{2}^{2} a_{3}^{2} a_{4}^{2}\right)$, is shown in Figure 3.

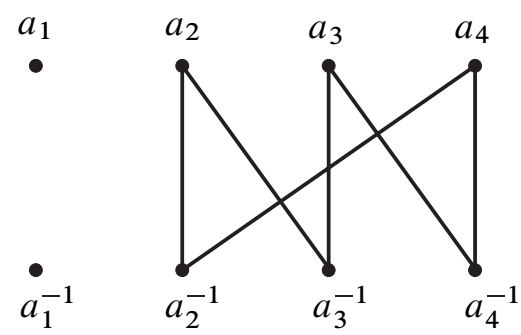

Figure 3. Augmented Whitehead graph $\widehat{\Gamma}_{\left\{a_{1}, a_{2}, a_{3}, a_{4}\right\}}\left(a_{2}^{2} a_{3}^{2} a_{4}^{2}\right)$.

Definition 4.2 (cut vertex). A cut vertex $v$ of a graph $\Gamma$ is a vertex such that $\Gamma=$ $\Gamma_{1} \cup \Gamma_{2}$, where $\Gamma_{1}$ and $\Gamma_{2}$ are nonempty subgraphs and $\Gamma_{1} \cap \Gamma_{2}=\{v\}$. If $\Gamma$ is disconnected, then all of its vertices are cut vertices. 
Whitehead proved [30] that the augmented Whitehead graph of a basis of a free group has a cut vertex. Note that a power of a primitive has the same augmented Whitehead graph as the given primitive, so the augmented Whitehead graph of a power of a primitive must also have a cut vertex. The converse is, of course, not true - for example, $a b a^{3} b$ is not primitive in $F_{2}$ - but of course the contrapositive is: having an augmented Whitehead graph with no cut vertex implies the element is not a primitive or a power of a primitive.

For our purposes, we will need a generalization of Whitehead's theorem due to Stallings [28], so we state it now. A subset $S$ of $F_{n}$ is called separable if there is a free factorization of $F_{n}$ with two factors such that each element of $S$ can be conjugated into one of the factors. In particular, a set is separable if its elements can be conjugated (possibly by different conjugators) to the elements of some basis of $F_{n}$. Thus, a basis (and the cyclic reduction of a basis) is always separable.

Theorem 4.3 ([28]). If $\boldsymbol{x}$ is a separable set in $F_{n}$, then there is a cut vertex in $\widehat{\Gamma}_{\boldsymbol{a}}(\boldsymbol{x})$.

Now consider our motivating example of the distance $d$ between $v_{0}=\left[H *\left\langle a_{n}\right\rangle\right]$ and $v=\left[H *\left\langle a_{n} h\right\rangle\right]$ in $\mathcal{E} \delta_{n}^{1}$. Naïvely, we could hope that if we could break up $h$, considered as a reduced word, into $k$ subwords such that each subword had an augmented Whitehead graph with no cut vertex, then $d$ might be bounded from below by a function of $k$. However, it may not be the case that such a decomposition of $h$ 'breaks' $h$ in the places corresponding to the most efficient way of decomposing it as a product of powers of primitives: a given primitive might contribute to one or more of the subwords. But Whitehead's theorem does not say that the (augmented) Whitehead graph of any subword of a primitive will have a cut vertex. Indeed, a primitive element conjugated by an arbitrary word will still be primitive, and the only reason its augmented Whitehead graph will have a cut vertex will be from the single self-loop contributed by the last and first letters. If the primitive element is cyclically reduced, then we may claim that the (non-augmented or augmented) Whitehead graph of any subword will have a cut vertex, but not otherwise.

The notions of $i$-length are defined precisely to deal with this delicate effect of conjugation. Simple $i$-length ignores conjugation completely, looking only at the non-augmented Whitehead graph of a word and its subwords. Conjugate reduced $i$-length takes all possible conjugations of the subwords of a word into account. Full $i$-length then uses conjugate reduced $i$-length to measure the complexity of an entire basis.

We are almost ready to give the definitions of $i$-length, but we need one minor piece of notation to proceed.

Notation 4.4. Elements of $F_{n}$ are equivalence classes of words in the alphabet $\boldsymbol{a} \cup \boldsymbol{a}^{-1}$ under free reduction. For two words $w_{1}$ and $w_{2}$ in this alphabet, we write $w_{1}=w_{2}$ if they are equal as words, and $w_{1}={ }_{r} w_{2}$ if they are equal after free reduction, i.e. as elements of $F_{n}$. 
We now define and study the three notions of $i$-length, in the following three sections.

4.2. Simple $i$-length. The definitions of conjugate reduced and full $i$-length are based on the straightforward notion of simple $i$-length. Simple $i$-length records the maximal number of pieces a word can be broken into such that the Whitehead graph of each piece has no cut vertex.

Definition 4.5 (Simple $i$-length). Fix an index $i \in\{1, \ldots, n\}$. Let $w$ be a word which contains no occurrence of $a_{i}^{ \pm 1}$. The simple $i$-length of $w$, denoted $|w|_{i}^{\text {simple }}$, is the greatest number $t$ such that $w$ is of the form $w_{1} w_{2} \ldots w_{t}$, where $\Gamma_{\boldsymbol{a}-\left\{a_{i}\right\}}\left(w_{j}\right)$ has no cut vertex for each $j=1, \ldots, t$. If $\Gamma_{\boldsymbol{a}-\left\{a_{i}\right\}}(w)$ has a cut vertex, we define $|w|_{i}^{\text {simple }}$ to be zero.

For example, for $w=a_{1}^{2} a_{2}^{2} \ldots a_{n-1}^{2} a_{1}$, we get $|w|_{n}^{\text {simple }}=1$ because $\Gamma_{\boldsymbol{a}-\left\{a_{i}\right\}}(w)$ has no cut vertex (so $|w|_{i}^{\text {simple }} \geq 1$ ), but if $w=w_{1} w_{2} \ldots w_{t}$ for nontrivial $w_{j}$ and $t>1$, then $\Gamma_{\boldsymbol{a}-\left\{a_{i}\right\}}\left(w_{j}\right)$ will have a cut vertex for at least one $j$ (so $|w|_{i}^{\text {simple }}<2$ ).

It worth pointing out that in the above definition we use standard Whitehead graph, not the augmented one.

Simple $i$-length has the following three useful properties, which we will use in future proofs.

Lemma 4.6. Let $w$ be a freely reduced word in $F_{n}$ which contains no occurrence of $a_{i}^{ \pm 1}$ and let $u$ and $v$ be disjoint subwords of $w$. Then

$$
|w|_{i}^{\text {simple }} \geq|u|_{i}^{\text {simple }}+|v|_{i}^{\text {simple }} .
$$

Proof. If $|u|_{i}^{\text {simple }}>0$ and $|v|_{i}^{\text {simple }}>0$ then consider the partitions of $u$ and $v$ into $|u|_{i}^{\text {simple }}$ and $|v|_{i}^{\text {simple }}$ pieces respectively. These partitions induce a partition of $w$ into $|u|_{i}^{\text {simple }}+|v|_{i}^{\text {simple }}$ pieces, where the portions of $w$ disjoint from $u$ and $v$ are appended to the first and last pieces in the partitions of $u$ and $v$. Note that appending will not brake the fact that the Whitehead graph of a piece does not have a cut vertex, because $w$ is freely reduced. If $|u|_{i}^{\text {simple }}=0$ (respectively, $|v|_{i}^{\text {simple }}=0$ ) then we similarly form a partition of $w$ into $|v|_{i}^{\text {simple }}$ (respectively, $|u|_{i}^{\text {simple }}$ ) pieces. In the case $|u|_{i}^{\text {simple }}=0$ and $|v|_{i}^{\text {simple }}=0$ the claim is trivial.

Lemma 4.7. Let $u$ and $v$ be freely reduced words which contain no occurrence of $a_{i}^{ \pm 1}$ such that $w=u v$ is freely reduced. Then

$$
|w|_{i}^{\text {simple }} \leq|u|_{i}^{\text {simple }}+|v|_{i}^{\text {simple }}+1 .
$$


Proof. If $|w|_{i}^{\text {simple }}=0$ then the claim is trivial. Otherwise let $w=w_{1} w_{2} \cdots w_{k}$ be a partition of $w$ realizing the simple $i$-length of $w$, and let $j$ denote the first index such that $w_{j}$ is not fully contained in $u$. This gives partitions $u=w_{1} w_{2} \cdots\left(w_{j-1} w_{j}^{\prime}\right)$ and $v=\left(w_{j}^{\prime \prime} w_{j+1}\right) w_{j+2} \cdots w_{k}$ of $u$ and $v$ showing that

$$
|u|_{i}^{\text {simple }}+|v|_{i}^{\text {simple }} \geq(j-1)+(k-j)=k-1=|w|_{i}^{\text {simple }}-1 .
$$

Lemma 4.8. If $w$ is a cyclically reduced word which contains no occurrence of $a_{i}^{ \pm 1}$ and $w^{\prime}$ is a cyclic conjugate of $w$, then

$$
|w|_{i}^{\text {simple }}-1 \leq\left|w^{\prime}\right|_{i}^{\text {simple }} \leq|w|_{i}^{\text {simple }}+1 .
$$

Proof. It is enough to show that cyclic conjugation cannot decrease the simple $i$ length by more than 1 . Let $\iota(w)$ be the initial segment of $w$ such that $w^{\prime}={ }_{r} w^{\iota(w)}$. Let $w=w_{1} w_{2} \cdots w_{k}$ be the partition of $w$ realizing the simple $i$-length of $w$, and let $j$ denote the first index such that $w_{j}$ is not fully contained in $\iota(w)$. Then $w^{\prime}$ can be partitioned as $\left(w_{j}^{\prime \prime} w_{j+1}\right) \ldots w_{k} w_{1} \ldots\left(w_{j-1} w_{j}^{\prime}\right)$ where $w_{j}=w_{j}^{\prime} w_{j}^{\prime \prime}$. Thus, $w^{\prime}$ can be partitioned into at least $k-1$ subwords of nontrivial simple $i$-length, and the lemma follows.

4.3. Conjugate reduced $i$-length. Conjugate reduced $i$-length is based on simple $i$-length, but additionally takes conjugation into account.

Definition 4.9 (Conjugate reduced $i$-length). Fix an index $i \in\{1, \ldots, n\}$. Let $w$ be a word which contains no occurrence of $a_{i}^{ \pm 1}$ (thought of as a subword of another word in the alphabet $\boldsymbol{a}^{ \pm 1}$ ). Then $w$ has conjugate reduced $i$-length at most $k$ if there exist freely reduced words $v_{1}, \ldots, v_{l}, u_{1}, \ldots, u_{l}$ such that:

(1) $w={ }_{r} v_{1}^{u_{1}} v_{2}^{u_{2}} \ldots v_{l}^{u_{l}}$, where $v_{j}^{u_{j}}:=u_{j}^{-1} v_{j} u_{j}$, and

(2) $k=(l-1)+\left|v_{1}\right|_{i}^{\text {simple }}+\cdots+\left|v_{l}\right|_{i}^{\text {simple }}$.

The decomposition of $w$ as $v_{1}^{u_{1}} v_{2}^{u_{2}} \ldots v_{l}^{u_{l}}$ is called a decomposition, and $k$ is the conjugate reduced $i$-length associated to the decomposition. If the associated $k$ is minimal among all such decompositions, the decomposition is called optimal, and $k$ is called a conjugate reduced $i$-length of $w$ and denoted by $|w|_{i}^{\text {cr }}$. The number $l$ of factors of the form $v_{j}^{u_{j}}$ in the decomposition is called the factor length of the decomposition.

We now wish to describe some properties of conjugate reduced $i$-length. However, before we do so, we need to verify that conjugate reduced $i$-length is not a trivial notion of complexity. In this section, we show that there exist words of arbitrary conjugate reduced $i$-length. In the process, we develop a useful lemma for working with $i$-length. Then, we collect four short lemmas which describe how conjugate reduced $i$-length is related to simple $i$-length, and how conjugate reduced $i$-length behaves under conjugation and multiplication. 
Definition 4.10 (canceling pairs). Let $w \in F_{n}$ be arbitrary reduced word which contains no occurrence of $a_{i}^{ \pm 1}$. A set of any two subwords of $w$ of the form $u$, $u^{-1}$ is called a canceling pair in $w$. A family $\mathcal{F}$ of canceling pairs in $w$ is called nested if canceling pairs in $\mathscr{F}$ are disjoint and, for any canceling pairs $u, u^{-1}$ and $v, v^{-1}$ in $\mathcal{F}, v$ occurs between $u$ and $u^{-1}$ in $w$ if and only if $v^{-1}$ does. If $\mathscr{F}$ is a nested family of canceling pairs for $w$, we abuse notation and let $w-\mathscr{F}$ denote the set of subwords of $w$ which are maximal under inclusion and which do not intersect any element of any canceling pair in $\mathscr{F}$ as subwords of $w$. Finally, we define $|w-\mathcal{F}|_{i}^{\text {simple }}:=|\mathcal{F}|+\sum_{w^{\prime} \in(w-\mathscr{F})}\left|w^{\prime}\right|_{i}^{\text {simple }}$.

Lemma 4.11. Let $w \in F_{n}$ be a nontrivial reduced word which contains no occurrence of $a_{i}^{ \pm 1}$ and let $T$ be the set consisting of all nested families of canceling pairs of $w$. Then

$$
|w|_{i}^{\text {cr }} \geq \min _{\mathcal{F} \in T}\left(\max \left\{\frac{|\mathcal{F}|}{2}-1, \frac{1}{5}|w-\mathscr{F}|_{i}^{\text {simple }}-3\right\}\right) .
$$

Proof. Let $\gamma=v_{1}^{u_{1}} v_{2}^{u_{2}} \cdots v_{l}^{u_{l}}$ be an optimal decomposition of $w$ realizing its conjugate reduced $i$-length.

First of all, since $\gamma$ is optimal, we may assume that all $v_{j}$ are cyclically reduced (cyclic reduction of $v_{j}$ cannot increase the conjugate reduced $i$-length).

Now we will utilize the technique used in the proof of the van Kampen lemma (see, for example, [23]). The word $w$ represents a trivial element in the group defined by the presentation

$$
\left\langle\boldsymbol{a} \mid v_{1}, v_{2}, \ldots, v_{l}\right\rangle .
$$

Consider the van Kampen diagram $\Gamma_{0}$ with boundary label $\gamma$ over the presentation (1) as depicted in Figure 4. This diagram is a wedge of $l$ "lollipops" corresponding to $l$ factors of $\gamma$ with "stems" labeled by the $u_{j}$ and with the "candies" (boundaries of 2-cells) labelled by the $v_{j}$. The base-vertex in $\Gamma_{0}$ is the common vertex of "lollipops".

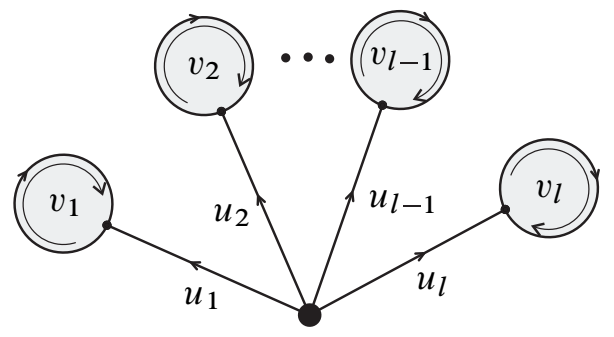

Figure 4. Van Kampen diagram $\Gamma_{0}$ corresponding to decomposition $\gamma$.

Fix some free reduction process transforming $\gamma$ to $w$. The $j$ th step of this reduction process takes the van Kampen diagram $\Gamma_{j-1}$ to the diagram $\Gamma_{j}$, and corresponds to modifying a pair of adjacent, inversely labeled edges along the boundary cycle of 
$\Gamma_{j-1}$. This has the effect of 'removing' this pair of edges from the boundary cycle of $\Gamma_{j}$ in the following sense. If these two edges have just one vertex in common, they are folded and if this common vertex has degree 2 in $\Gamma_{j-1}$ then the edge obtained by folding is removed from $\Gamma_{j-1}$. If they have two vertices in common, the union of 2 -cells bounded by these two edges is completely removed from $\Gamma_{j-1}$. This folding or removing defines a new van Kampen diagram $\Gamma_{j}$. At the end of the process we obtain a van Kampen diagram $\Gamma$ with boundary label $w$ shown in Figure 5. Note that in the reduction process the number of 2-cells in each successive van Kampen diagram does not grow, so the number $l^{\prime}$ of 2-cells in $\Gamma$ does not exceed $l$.

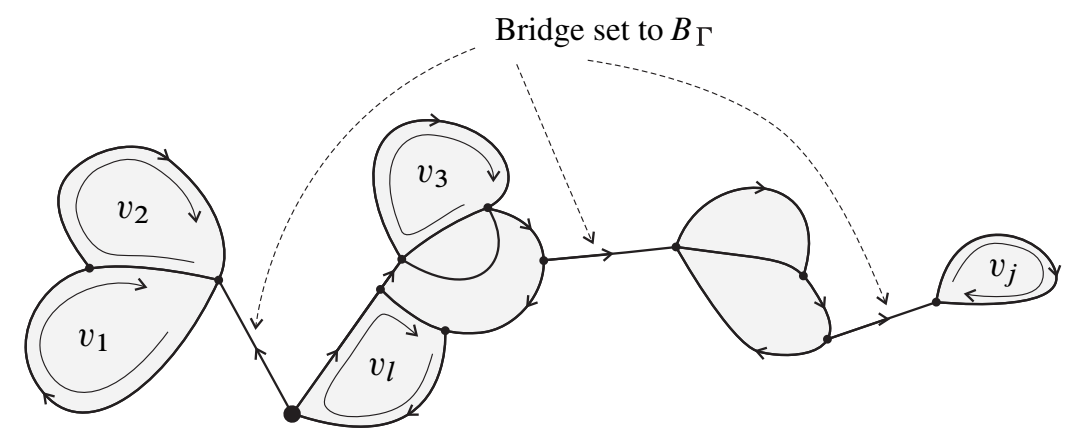

Figure 5. Van Kampen diagram $\Gamma$ after folding.

Because each of the $v_{j}$ 's is cyclically reduced, the boundary of each 2-cell in the diagram $\Gamma$ is labeled by a cyclic conjugate of $v_{j}$ that depends on where along the boundary one begins reading. The bridge set $B_{\Gamma}$ of $\Gamma$ is the set of all vertices and edges whose deletion from the topological realization $|\Gamma|$ of $\Gamma$ would disconnect it. A disk-component of $\Gamma$ is a subset of $\Gamma$ which is the closure of a connected component of $|\Gamma|-\left|B_{\Gamma}\right|$. The disk-components of $\Gamma$ are joined by (possibly trivial) edge-paths from the bridge set. Retracting each of these paths to a point produces a new van Kampen diagram $\Gamma^{\prime}$ with a boundary label $u$ obtained from $w$ by removing a nested family of canceling pairs, denoted $\mathcal{F}$, where each canceling pair corresponds to a path inside $B_{\Gamma}$ whose inner vertices have degree 2. Such a diagram is depicted in Figure 6. Note that $u$ is not necessarily freely reduced, but that $u$ is the product of subwords in $w-\mathcal{F}$, all of which are subwords of $w$ and hence freely reduced. The vertices of degree at least three along the boundary of $\Gamma^{\prime}$ split $u$ into subwords $w_{1}, w_{2}, \ldots, w_{k}$, where each $w_{j}$ is a part of the boundary of a 2-cell in $\Gamma$. This partition of $u$ refines the partition $w_{1}^{\prime}, w_{2}^{\prime}, \ldots, w_{r}^{\prime}$ of $u$ induced by $w-\mathcal{F}$.

Collapsing all disc components of $\Gamma$ and removing vertices of degree 2 leaves the tree with $e$ edges and $r^{\prime}$ vertices of degree 1 , each of which was obtained by collapsing one of the disc components. In every such tree we have $e \leq 2 r^{\prime}$. For the number of canceling pairs in $\mathcal{F}$ we get $|\mathcal{F}|=e+r^{\prime \prime}$, where $r^{\prime \prime}$ is the number of disc components in $\Gamma$ that collapse to the vertices of degree 2 . But since each disc 


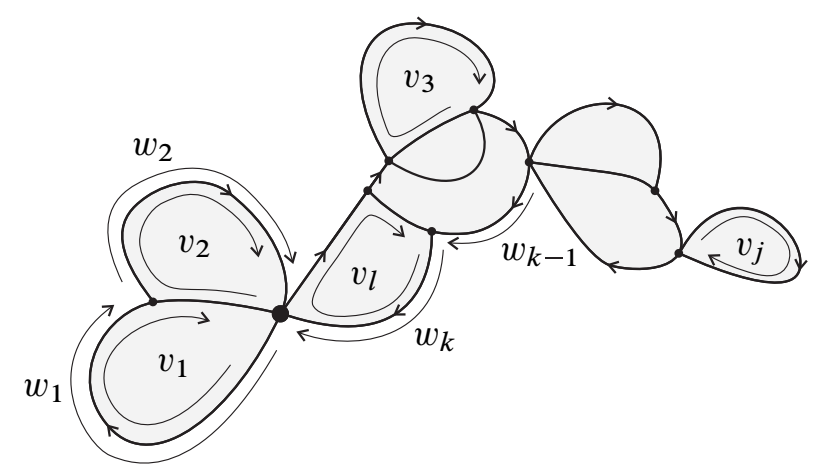

Figure 6. Van Kampen diagram $\Gamma^{\prime}$ after bridge retraction.

component produces at least one $w_{j}^{\prime}$, we get

$$
|\mathcal{F}|=e+r^{\prime \prime} \leq 2 r^{\prime}+r^{\prime \prime} \leq 2\left(r^{\prime}+r^{\prime \prime}\right) \leq 2 r
$$

We have by Lemma 4.7 and the last inequality that

$$
\begin{aligned}
|w-\mathcal{F}|_{i}^{\text {simple }} & =|\mathcal{F}|+\sum_{j=1}^{r}\left|w_{j}^{\prime}\right|_{i}^{\text {simple }} \leq 2 r+\sum_{j=1}^{k}\left|w_{j}\right|_{i}^{\text {simple }}+(k-r) \\
& =k+r+\sum_{j=1}^{k}\left|w_{j}\right|_{i}^{\text {simple }} .
\end{aligned}
$$

By construction each $w_{j}$ is a subword of a cyclic conjugate $v_{t}^{\prime}$ of some $v_{t}$ representing the label of the boundary of 2-cell in $\Gamma^{\prime}$ to which $w_{j}$ belongs. It may happen that several $w_{j}$ lie on the boundary of one cell labelled by a conjugate of $v_{t}$, but by construction these occurrences do not overlap. Let $\left\{c_{1}, \ldots, c_{l}\right\}$ denote the set of 2-cells in $\Gamma_{0}$. Denote by $\tilde{c}_{t}$ the image of a cell $c_{t}$ in $\Gamma^{\prime}$ and assume that $v_{j}$ is a boundary label of $\tilde{c}_{j}$ (that, of course, coincides with a boundary label of $c_{j}$ ). Then the sum in (3) can be rewritten as

$$
|w-\mathcal{F}|_{i}^{\text {simple }} \leq k+r+\sum_{t=1}^{l} \sum_{w_{j} \in \partial \tilde{c}_{t}}\left|w_{j}\right|_{i}^{\text {simple }}
$$

where if two or more 2-cells in $\Gamma_{0}$ are mapped to the same cell in $\Gamma^{\prime}$, then we simply add extra terms to the righthand side of 4 not jeopardizing the inequality. Since by Lemmas 4.6 and 4.8,

$$
\sum_{w_{j} \in \partial \tilde{c}_{t}}\left|w_{j}\right|_{i}^{\text {simple }} \leq\left|v_{t}^{\prime}\right|_{i}^{\text {simple }} \leq\left|v_{t}\right|_{i}^{\text {simple }}+1
$$


we can transform inequality (4) to

$$
|w-\mathcal{F}|_{i}^{\text {simple }} \leq k+r+\sum_{t=1}^{l}\left(\left|v_{t}\right|_{i}^{\text {simple }}+1\right)=|w|_{i}^{\text {cr }}+k+r+1 \leq|w|_{i}^{\text {cr }}+2 k+1
$$

To finish the proof of the lemma we first prove that $k \leq 2 l^{\prime}-1 \leq 2 l-1$. This follows by induction on the number $l^{\prime}$ of cells of $\Gamma$ as follows. Clearly if $l^{\prime}=1$ then $k=1$. Assume that for any bridge-free van Kampen diagram with $l^{\prime}-12$-cells the number of arcs along the boundary without vertices of degree at least 3 is at most $2\left(l^{\prime}-1\right)-1$. Choose a 2 -cell $c$ in $\Gamma$ whose boundary contains a piece $p$ of boundary of $\Gamma$ and such that after $p$ and interior of $c$ are removed from $\Gamma$ the resulting diagram $\Gamma-p$ is still bridge-free and connected. There are several cases describing how $p$ may be attached to the boundary of $\Gamma-p$. It is straightforward to check that, in all cases, the attaching of $p$ can increase the number of arcs without vertices of degree at least 3 by at most 2 .

Finally, consider two cases. If $|w-\mathcal{F}|_{i}^{\text {simple }} \leq 5 l-5$, then

$$
|w|_{i}^{\mathrm{cr}} \geq l-1 \geq \frac{1}{5}|w-\mathcal{F}|_{i}^{\text {simple }}
$$

But if $|w-\mathcal{F}|_{i}^{\text {simple }}>5 l-5$, then since $k \leq 2 l-1<\frac{2}{5}|w-\mathcal{F}|_{i}^{\text {simple }}+1$ we get from (5)

$$
|w|_{i}^{\mathrm{cr}} \geq|w-\mathcal{F}|_{i}^{\text {simple }}-2 k-1>\frac{1}{5}|w-\mathcal{F}|_{i}^{\text {simple }}-3 .
$$

This proves half of the lemma.

For the second part of the lemma note that by 2

$$
|\mathcal{F}| \leq 2\left(r^{\prime}+r^{\prime \prime}\right) \leq 2 l
$$

since $r^{\prime}+r^{\prime \prime}$ does not exceed the number of all disk components in $\Gamma$ and each disk component contains at least one cell. Thus,

$$
|w|_{i}^{\mathrm{cr}} \geq l-1 \geq \frac{|\mathcal{F}|}{2}-1
$$

The statement of the lemma now follows.

Corollary 4.12. If $w$ is a positive word, then

$$
|w|^{\mathrm{cr}} \geq \frac{1}{5}|w|^{\text {simple }}-3
$$

Proof. If $w$ is positive, then the only possible family of canceling pairs is the trivial family. 
Note, that it's not true that simple and conjugate reduced $i$-lengths always coincide even for positive words. For example, for $w=a_{1}^{2} a_{2}^{2} \ldots a_{n-1}^{2} a_{1}$ we have $|w|_{i}^{\text {simple }}=1$, but $|w|_{i}^{\text {cr }}=0$ since $w=\left(a_{1}^{3} a_{2}^{2} \ldots a_{n-1}^{2}\right)^{a_{1}}$ and $\left|a_{1}^{3} a_{2}^{2} \ldots a_{n-1}^{2}\right|_{i}^{\text {simple }}=0$ because the Whitehead graph of $a_{1}^{3} a_{2}^{2} \ldots a_{n-1}^{2}$ has a cut vertex. However, it might be the case that this is about all we can do and for positive words $|w|_{i}^{\text {cr }} \geq|w|_{i}^{\text {simple }}-1$. The weaker observation above is enough for our purposes.

Corollary 4.13. There exist words of arbitrary (simple, conjugate reduced) $i$-length.

Proof. It now follows from the previous corollary that, for $w=a_{1}^{2} a_{2}^{2} \ldots a_{n-1}^{2} a_{1}$,

$$
\left|w^{l}\right|_{n}^{\mathrm{cr}} \geq l / 5-3 .
$$

We now state prove some properties of the conjugate reduced $i$-length.

Lemma 4.14. Conjugate reduced $i$-length is invariant under conjugation.

Proof. Let $w$ and $u$ be words that do not involve $a_{i}$, so that $w^{u}$ is a conjugate of $w$. Then there is a one-to-one correspondence between decompositions of $w$ and decompositions of $w^{u}$ defined by

$$
w={ }_{r} v_{1}^{u_{1}} v_{2}^{u_{2}} \ldots v_{l}^{u_{l}} \leftrightarrow v_{1}^{u_{1} u} v_{2}^{u_{2} u} \ldots v_{l}^{u_{l} u}={ }_{r} w^{u} .
$$

Now the statement of the lemma follows from the definition of conjugate reduced $i$-length.

The following lemma relates simple and conjugate reduced $i$-length.

Lemma 4.15. For any reduced word $w$,

$$
|w|_{i}^{\text {simple }} \geq|w|_{i}^{\mathrm{cr}} .
$$

If $|w|_{i}^{\text {cr }}>0$, then the Whitehead graph $\Gamma_{\boldsymbol{a}-\left\{a_{i}\right\}}(w)$ has no cut vertex.

Proof. A word $w$ represents a decomposition of itself with one factor whose conjugate reduced $i$-length is equal by definition to $|w|_{i}^{\text {simple }}$.

If $|w|_{i}^{\text {cr }}>0$, then $|w|_{i}^{\text {simple }}>0$. If the Whitehead graph $\Gamma_{\boldsymbol{a}-\left\{a_{i}\right\}}(w)$ had a cut vertex, then since $w$ is freely reduced the Whitehead graph of any subword of $w$ would also have a cut vertex. This contradicts that $|w|_{i}^{\text {simple }}>0$.

Finally, we have the two lemmas describing how conjugate reduced $i$-length behaves under multiplication.

Lemma 4.16. For any words $u$ and $v$, we have

$$
|u|_{i}^{\mathrm{cr}}-|v|_{i}^{\mathrm{cr}}-1 \leq|u v|_{i}^{\mathrm{cr}} \leq|u|_{i}^{\mathrm{cr}}+|v|_{i}^{\mathrm{cr}}+1 .
$$


Proof. A decomposition of $u v$ may be obtained by concatenating optimal decompositions of $u$ and $v$. The associated $i$-length of this decomposition of $u v$ yields the second inequality. The first inequality follows from the second inequality by concatenating $u v$ and $v^{-1}:|u|_{i}^{\text {cr }}=\left|u v v^{-1}\right|_{i}^{\text {cr }} \leq|u v|_{i}^{\text {cr }}+|v|_{i}^{\text {cr }}+1$.

Thanks to the referee for pointing out a simplified proof of the following lemma.

Lemma 4.17. For any words $u$, $v$ and $w$, we have

$$
|u v|_{i}^{\mathrm{cr}}-|w|_{i}^{\mathrm{cr}}-1 \leq|u w v|_{i}^{\mathrm{cr}} \leq|u v|_{i}^{\mathrm{cr}}+|w|_{i}^{\mathrm{cr}}+1 .
$$

Proof. As conjugate reduced $i$-length is invariant under conjugation by Lemma 4.14, we get $|u w v|_{i}^{\text {cr }}=|v u w|_{i}^{\text {cr }}$ and $|v u|_{i}^{\text {cr }}=|u v|_{i}^{\text {cr }}$. The lemma then follows from Lemma 4.16.

4.4. Full $i$-length. We are now ready to define the (full) $i$-length of a basis for $F_{n}$ (or more generally a set of words). Given a basis $Y$, we essentially measure the maximal conjugate reduced $i$-length of any subword of any element of $Y$. However, we must be very careful to properly account for conjugation. We do so as follows.

Let $\boldsymbol{y}$ be a set of reduced words in the alphabet $\boldsymbol{a}^{ \pm 1}$. Let $\tilde{\boldsymbol{y}}$ denote the set of elements of $\boldsymbol{y}$ after each of them has been cyclically reduced. Define $w_{L}=w_{L}(\boldsymbol{y})$ to be the longest word in the alphabet $\left(\boldsymbol{a}-\left\{a_{i}\right\}\right)^{ \pm 1}$ such that every occurrence of $a_{i}$ in every $\tilde{y} \in \tilde{\boldsymbol{y}}$ is cyclically preceded by $w_{L}$ and every occurrence of $a_{i}^{-1}$ is cyclically followed by $w_{L}^{-1}$ (note $w_{L}$ could be trivial). Similarly, let $w_{R}=w_{R}(\boldsymbol{y})$ be the longest word in $\left(\boldsymbol{a}-\left\{a_{i}\right\}\right)^{ \pm 1}$ such that every occurrence of $a_{i}$ in every $\tilde{y} \in \tilde{\boldsymbol{y}}$ is cyclically followed by $w_{R}$, every occurrence of $a_{i}^{-1}$ is cyclically preceded by $w_{R}^{-1}$, and no such occurrence of $w_{R}$ intersects any such occurrence of $w_{L}$ (again, $w_{R}$ could be trivial). Let $\alpha^{\prime}=\alpha_{y}^{\prime}$ be the automorphism of $F_{n}$ which maps $a_{i}$ to $w_{L}^{-1} a_{i} w_{R}^{-1}$. Let $w_{C}=w_{C}(\boldsymbol{y})$ be the longest word in $\left(\boldsymbol{a}-\left\{a_{i}\right\}\right)^{ \pm 1}$ such that, in $\alpha^{\prime} \tilde{\boldsymbol{y}}$, every occurrence of $a_{i}^{k}$ either: (a) occurs by itself as an element of $\alpha^{\prime} \tilde{\boldsymbol{y}}$ or (b) appears cyclically conjugated by $w_{C}$, so that $a_{i}^{k}$ is cyclically preceded by $w_{C}^{-1}$ and cyclically followed by $w_{C}$. If every occurrence of $a_{i}^{k}$ occurs by itself, we declare that $w_{C}$ is trivial.

If $y$ is a singleton $\{y\}$, we abuse notation and write $y$ instead of $\{y\}$ when applying any function in this subsection.

Let $\alpha=\alpha_{y}$ be the automorphism of $F_{n}$ which maps $a_{i}$ to $w_{L}^{-1} w_{C} a_{i} w_{C}^{-1} w_{R}^{-1}$. Thus, $w_{L}(\alpha \boldsymbol{y})=w_{R}(\alpha \boldsymbol{y})=1$ are trivial. The preimage of $a_{i}^{k}$ under $\alpha$, after free reduction of occurrences of $w_{C}^{-1} w_{C}$, is $w_{C}^{-1}\left(w_{L} a_{i} w_{R}\right)^{k} w_{C}$. Note $\alpha \boldsymbol{y}$ may not be cyclically reduced.

An $i$-chunk of a word $y$ in the alphabet $\boldsymbol{a}^{ \pm 1}$ is a cyclic subword of $\tilde{y}$ (here again, $\tilde{y}$ denotes the result of a cyclic reduction of $y$ ) which contains no $a_{i}^{ \pm 1}$ and is maximal among such subwords ordered by inclusion. By definition, every $i$-chunk of $y$ begins with either $w_{R}(y)$ or $\left(w_{L}(y)\right)^{-1}$, and ends with either $w_{L}(y)$ or $\left(w_{R}(y)\right)^{-1}$. 
For example, in the set $\boldsymbol{y}=\left\{a_{2}^{-1} a_{3} a_{4} a_{1} a_{4} a_{2} a_{3}, a_{4}^{-1} a_{1}^{-1} a_{4}^{-1} a_{3}^{-1}\right\}$, we have $\tilde{\boldsymbol{y}}=\boldsymbol{y}$. For $i=1, w_{L}(\boldsymbol{y})=a_{3} a_{4}, w_{R}(\boldsymbol{y})=a_{4}$, and $w_{C}(\boldsymbol{y})=a_{2}$. Thus, $\alpha\left(a_{1}\right)=a_{4}^{-1} a_{3}^{-1} a_{2} a_{1} a_{2}^{-1} a_{4}^{-1}$, so that $\alpha(y)=\left\{a_{1} a_{3}, a_{2} a_{1}^{-1} a_{2}^{-1}\right\}$.

Definition 4.18 (Full $i$-length). Fix an index $i \in\{1, \ldots, n\}$. Let $\boldsymbol{y}$ be a set of words in the alphabet $\boldsymbol{a}^{ \pm 1}$. The (full) $i$-length of $\boldsymbol{y}$ is

$$
|\boldsymbol{y}|_{i}:=\left|w_{R}(\boldsymbol{y}) w_{L}(\boldsymbol{y})\right|_{i}^{\text {cr }} .
$$

The following corollary immediately follows from the above definition and Corollary 4.13 .

Corollary 4.19. There exist bases of $F_{n}$ of arbitrary full $i$-length.

Proof. Consider a basis

$$
\boldsymbol{x}=\left(a_{1}, a_{2}, \ldots, a_{n-1}, a_{n} w^{l}\right)
$$

of $F_{n}$, where $w=a_{1}^{2} a_{2}^{2} \ldots a_{n-1}^{2} a_{1}$. Then, by Corollary 4.13,

$$
|\boldsymbol{x}|_{n}=\left|w^{l}\right|_{n}^{\text {cr }} \geq l / 5-3 .
$$

We now consider properties of full $i$-length. The key observation for our paper is the following lemma.

Lemma 4.20. For any basis $\boldsymbol{x}$ of $F_{n}$, any $x \in \boldsymbol{x}$, and any subword $w$ of an $i$-chunk of $\alpha_{\boldsymbol{x}} x$, we have $|w|_{i}^{\mathrm{cr}}=0$.

It follows that this result holds for any subset of any basis as well.

Proof. Throughout this proof, for sake of simplicity of notation, we write $\alpha$ for $\alpha_{\boldsymbol{x}}$. As $\boldsymbol{x}$ is a basis, so is $\alpha \boldsymbol{x}$. By definition, the full $i$-length of an element or of a set of elements is invariant under conjugation, where we may even conjugate different elements in the set by different conjugators. Therefore cyclic reduction of all elements of $\alpha \boldsymbol{x}$ does not change any $i$-length involved. Let $\boldsymbol{y}$ be the set $\widetilde{\alpha \boldsymbol{x}}$ obtained from $\alpha \boldsymbol{x}$ by cyclically reducing every element. Since $\alpha \boldsymbol{x}$ is a basis, $\boldsymbol{y}$ is a separable set. Therefore by Theorem 4.3 the augmented Whitehead graph $\widehat{\Gamma}_{\boldsymbol{a}}(\boldsymbol{y})$ of $\boldsymbol{y}$ has a cut vertex. Note that this graph does not have vertex loops since each word in $\boldsymbol{y}$ is cyclically reduced.

Proof by contradiction: assume that there exists some subword $w$ of an $i$-chunk of $\alpha x$ with $|w|_{i}^{\text {cr }}>0$. As $|w|_{i}^{\text {cr }}>0$, by Lemma 4.15 , the subgraph $\Gamma^{\prime}$ of $\widehat{\Gamma}_{\boldsymbol{a}}(\boldsymbol{y})$ on the vertex set corresponding to $\boldsymbol{a}-\left\{a_{i}\right\}$ has no cut vertex (since there are no vertex loops in the graph). It remains to consider the vertices corresponding to $a_{i}^{ \pm 1}$ in $\widehat{\Gamma}_{\boldsymbol{a}}(\boldsymbol{y})$. Since $a_{i}$ must appear as a letter in $\boldsymbol{x}$ and, hence, in $\boldsymbol{y}$, by the definition of Whitehead graph each of $a_{i}, a_{i}^{-1}$ has at least one neighbor in $\widehat{\Gamma}_{\boldsymbol{a}}(\boldsymbol{y})$. 
Consider the case when either $a_{i}$ or $a_{i}^{-1}$ has exactly one neighbor in $\hat{\Gamma}_{\boldsymbol{a}}(\boldsymbol{y})$. Without loss of generality, assume $a_{i}$ has exactly one neighbor. If the neighbor $b$ of $a_{i}$ were in $\Gamma^{\prime}$, we would contradict the definition of $w_{R}(\boldsymbol{x}): b^{-1}$ should have been appended to $w_{R}(\boldsymbol{x})$.

Thus, the only neighbor of $a_{i}$ must be $a_{i}^{-1}$. In this case, each occurrence of $a$ (resp. $a^{-1}$ ) in $\alpha \boldsymbol{x}$ must be cyclically followed (resp. preceded) by $a$ (resp. $a^{-1}$ ). The only way for this to occur is if every element of $\boldsymbol{y}$ involving $a_{i}$ is some power of $a_{i}$. But elements of $\boldsymbol{y}$ are primitives in $F_{n}$ as they are conjugates of basis elements of $\alpha \boldsymbol{x}$. Therefore this power can only be $a_{i}^{ \pm 1}$. Moreover, if there are two elements in $\boldsymbol{y}$ of the form $a_{i}^{ \pm 1}$, then there should be two conjugates of $a$ or $a^{-1}$ in $\alpha \boldsymbol{x}$, which is impossible because in this case we can obtain a commutator as a primitive element of $F_{n}$. Thus, we may assume without loss of generality that $a_{i}$ is an element of $\boldsymbol{y}$ and no other element of $\boldsymbol{y}$ contains an occurrence of $a_{i}$.

Since $\boldsymbol{y}$ was obtained from $\alpha \boldsymbol{x}$ by conjugating its elements, the structure of $\alpha \boldsymbol{x}$ is as follows. There is one element of the form $a_{i}^{w}$ for some $w \in F_{n}$, whose conjugate in $\boldsymbol{y}$ is $a_{i}$. All other elements in $\alpha \boldsymbol{x}$ are conjugates of words in $\boldsymbol{y}$ not involving $a_{i}$ by conjugators that may generally contain $a_{i}$. Then $z=(\alpha \boldsymbol{x})^{w^{-1}}$ is a basis for $F_{n}$ one of whose elements is $a_{i}$ and the others are conjugates of words in $\boldsymbol{y}$ where the words in $\boldsymbol{y}$ do not involve $a_{i}$ (but the conjugators could).

By Proposition 2.3 there is a sequence $\left(\delta_{j}\right), 1 \leq j \leq t$ of elementary Nielsen transformations taking $z$ to the standard basis $\boldsymbol{a}$ obtained from the Nielsen reduction process. In other words,

$$
(z)\left(\prod_{j=1}^{t} \delta_{j}\right)=a .
$$

Since the Nielsen reduction process does not increase the length of basis elements, the element $a_{i}$ in $z$ will be invariant under each transvection $\delta_{j}$. Let $S=\{j$ : $\delta_{j}$ does not involve $\left.a_{i}\right\}$ and consider the basis

$$
\boldsymbol{u}=(\boldsymbol{a})\left(\prod_{j \in S} \delta_{j}\right)^{-1}
$$

for $F_{n}$. By construction this basis is obtained from $z$ by removing all occurrences of $a_{i}$ from $z$ except a single occurrence of $a_{i}$ as an element of $z$. This implies that all other elements of $\boldsymbol{u}$ form a basis for $\left\langle\boldsymbol{a}-\left\{a_{i}\right\}\right\rangle$. On the other hand, elements of the basis $\boldsymbol{u}$ are conjugates of elements of $\boldsymbol{z}$. Therefore cyclic reduction of elements in $\boldsymbol{u}$ gives the set $\boldsymbol{y}$ up to cyclic conjugation. But then $\boldsymbol{y}-\left\{a_{i}\right\}$ is a separable set in $\left\langle\boldsymbol{a}-\left\{a_{i}\right\}\right\rangle$, and is such that $\widehat{\Gamma}_{\boldsymbol{a}-\left\{a_{i}\right\}}\left(\boldsymbol{y}-\left\{a_{i}\right\}\right)$ has no cut vertex. This contradicts Theorem 4.3, and shows that neither $a_{i}$ nor $a_{i}^{-1}$ may have exactly one neighbor in $\widehat{\Gamma}_{\boldsymbol{a}}(\boldsymbol{y})$.

We are left to consider the remaining case, when both $a_{i}$ and $a_{i}^{-1}$ have at least two neighbors in $\widehat{\Gamma}_{\boldsymbol{a}}(\boldsymbol{y})$. As $\Gamma^{\prime}$ contains no cut vertex, the only way for $\widehat{\Gamma}_{\boldsymbol{a}}(\boldsymbol{y})$ to still have a cut vertex in this situation is if $a_{i}$ and $a_{i}^{-1}$ both have exactly two neighbors in 
$\hat{\Gamma}_{\boldsymbol{a}}(\boldsymbol{y})$, both are neighbors of each other, and both share a common third neighbor, say $b$. This means that every occurrence of $a_{i}^{k}, k \neq 0$, in $\alpha \boldsymbol{x}$ appears by itself in $\alpha \boldsymbol{x}$ or appears conjugated by $b^{-1}$. But this contradicts the definition of $w_{C}(\boldsymbol{x})$ : the letter $b^{-1}$ should have been appended to $w_{C}$.

As corollaries of the above lemma and definition of the $i$-length of a set we get the following statements.

Corollary 4.21. For any basis $\boldsymbol{x}$ of $F_{n}$, the maximal conjugate reduced $i$-length of an $i$-chunk of $\alpha_{\boldsymbol{x}} x$ over all elements $x \in \boldsymbol{x}$ is 0 .

Lemma 4.22. For any basis $\boldsymbol{x}$ and any $x \in \boldsymbol{x}$ containing $a_{i}$,

$$
|\boldsymbol{x}|_{i}-2 \leq|x|_{i} \leq|\boldsymbol{x}|_{i}+2 .
$$

Proof. By the definition of $\alpha_{\boldsymbol{x}}$, for any $x \in \boldsymbol{x},\left|\alpha_{\boldsymbol{x}} x\right|_{i}^{\text {cr }}=0$. Without loss of generality, as $i$-length is unaffected by conjugation assume that $x$ is such that all of $x, \alpha_{\boldsymbol{x}}^{\prime} x$, and $\alpha_{x} x$ are cyclically reduced. For simplicity of notation, let $\alpha^{\prime}:=\alpha_{x}^{\prime}$. Note that every occurrence of $a_{i}$ in $\alpha^{\prime} x$ occurs in a subword of $\alpha^{\prime} x$ in at least one of the following four forms: $a_{i}, w_{C}(\boldsymbol{x})^{-1} a_{i}, a_{i} w_{C}(\boldsymbol{x}), w_{C}(\boldsymbol{x})^{-1} a_{i} w_{C}(\boldsymbol{x})$. Similarly, every occurrence of $a_{i}^{-1}$ in $\alpha^{\prime} x$ occurs in a subword of $\alpha^{\prime} x$ in at least one of the forms: $a_{i}^{-1}, w_{C}(x)^{-1} a_{i}^{-1}$, $a_{i}^{-1} w_{C}(\boldsymbol{x}), w_{C}(\boldsymbol{x})^{-1} a_{i}^{-1} w_{C}(\boldsymbol{x})$.

Consider the following possible cases.

Case 1. The word $\alpha^{\prime} x$ is a power of $a_{i}$, so that no occurrence of $a_{i}$ (or its inverse) appears in $x$ multiplied by $w_{C}(\boldsymbol{x})$ (or its inverse). In this case $w_{L}(x)=$ $w_{R}(\boldsymbol{x}) w_{L}(\boldsymbol{x})$ and $w_{R}(x)$ is trivial, therefore $w_{R}(x) w_{L}(x)=w_{R}(\boldsymbol{x}) w_{L}(\boldsymbol{x})$ and $|x|_{i}=|\boldsymbol{x}|_{i}$.

Case 2. Some occurrences of $a_{i}$ (or its inverse) in $\alpha^{\prime} x$ occur in subwords of $\alpha^{\prime} x$ of the form $w_{C}(\boldsymbol{x})^{-1} a_{i} w_{C}(\boldsymbol{x})$ (resp. $w_{C}(\boldsymbol{x})^{-1} a_{i}^{-1} w_{C}(\boldsymbol{x})$ ), while some do not. Note that the last letter in $w_{C}(\boldsymbol{x})$ must differ from the last letter in $w_{R}(\boldsymbol{x})$ since these letters do not cancel in $w_{R}(\boldsymbol{x}) w_{C}(\boldsymbol{x})^{-1}$. But this implies that $w_{L}(x)=w_{L}(\boldsymbol{x})$ and $w_{R}(x)=w_{R}(\boldsymbol{x})$, again implying $|x|_{i}=|\boldsymbol{x}|_{i}$.

Case 3. Every $a_{i}$ (resp. $a_{i}^{-1}$ ) in $x$ occurs in $\alpha^{\prime} x$ in a subword of $\alpha^{\prime} x$ of the form $w_{C}(\boldsymbol{x})^{-1} a_{i} w_{C}(\boldsymbol{x})$ (resp. $w_{C}(\boldsymbol{x})^{-1} a_{i}^{-1} w_{C}(\boldsymbol{x})$ ). It follows that $w_{L}(x)$ contains $w_{C}(\boldsymbol{x})^{-1} w_{L}(\boldsymbol{x})$ as a terminal segment. It may also contain some portion $w_{2}$ of an $i$-chunk of $\alpha_{x}(x)$ of zero $i$-length by Lemma 4.20, and finally it may contain some portion of $w_{R}(\boldsymbol{x}) w_{C}(\boldsymbol{x})$. In any case $w_{R}(x)$ will contain the rest of $w_{R}(\boldsymbol{x}) w_{C}(\boldsymbol{x})$ and possibly some portion $w_{1}$ of an $i$-chunk of $\alpha_{\boldsymbol{x}}(x)$ also of zero $i$-length. Therefore

$$
w_{R}(x) w_{L}(x)=w_{R}(\boldsymbol{x}) w_{C}(\boldsymbol{x}) w_{1} w_{2} w_{C}(\boldsymbol{x})^{-1} w_{L}(\boldsymbol{x}),
$$


where $w_{1}$ and $w_{2}$ may be trivial. Therefore applying Lemmas 4.17 and 4.16, and taking into account that conjugation does not change the conjugate reduced $i$-length of a subword, we obtain

$$
\begin{aligned}
|x|_{i} & =\left|w_{R}(x) w_{L}(x)\right|_{i}^{\mathrm{cr}} \\
& =\left|w_{R}(\boldsymbol{x}) w_{C}(\boldsymbol{x})^{-1} w_{1} w_{2} w_{C}(\boldsymbol{x}) w_{L}(\boldsymbol{x})\right|_{i}^{\mathrm{cr}} \\
& \leq\left|w_{R}(\boldsymbol{x}) w_{L}(\boldsymbol{x})\right|_{i}^{\mathrm{cr}}+\left|w_{C}(\boldsymbol{x})^{-1} w_{1} w_{2} w_{C}(\boldsymbol{x})\right|_{i}^{\mathrm{cr}}+1 \\
& =\left|w_{R}(\boldsymbol{x}) w_{L}(\boldsymbol{x})\right|_{i}^{\mathrm{cr}}+\left|w_{1} w_{2}\right|_{i}^{\mathrm{cr}}+1 \\
& \leq\left|w_{R}(\boldsymbol{x}) w_{L}(\boldsymbol{x})\right|_{i}^{\mathrm{cr}}+\left|w_{1}\right|_{i}^{\mathrm{cr}}+\left|w_{2}\right|_{i}^{\mathrm{cr}}+2 \\
& =\left|w_{R}(\boldsymbol{x}) w_{L}(\boldsymbol{x})\right|_{i}^{\mathrm{cr}}+2 \\
& =|\boldsymbol{x}|_{i}+2 .
\end{aligned}
$$

Similarly we derive the first inequality

$$
\begin{aligned}
|\boldsymbol{x}|_{i} & =\left|w_{R}(\boldsymbol{x}) w_{L}(\boldsymbol{x})\right|_{i}^{\mathrm{cr}} \\
& =\left|w_{R}(\boldsymbol{x}) w_{C}(\boldsymbol{x})^{-1} w_{1} w_{2}\left(w_{1} w_{2}\right)^{-1} w_{C}(\boldsymbol{x}) w_{L}(\boldsymbol{x})\right|_{i}^{\mathrm{cr}} \\
& \leq\left|w_{R}(\boldsymbol{x}) w_{C}(\boldsymbol{x})^{-1} w_{1} w_{2} w_{C}(\boldsymbol{x}) w_{L}(\boldsymbol{x})\right|_{i}^{\mathrm{cr}}+\left|w_{1} w_{2}\right|_{i}^{\mathrm{cr}}+1 \\
& \leq\left|w_{R}(x) w_{L}(x)\right|_{i}^{\mathrm{cr}}+\left|w_{1}\right|_{i}^{\mathrm{cr}}+\left|w_{2}\right|_{i}^{\mathrm{cr}}+2 \\
& =\left|w_{R}(x) w_{L}(x)\right|_{i}^{\mathrm{cr}}+2 \\
& =|x|_{i}+2 .
\end{aligned}
$$

Note that the last case covers all situations when $w_{C}(\boldsymbol{x})$ is trivial.

As an immediate corollary we obtain the following statement, which is a basis for distance estimates in Section 5.

Corollary 4.23. For any bases $\boldsymbol{x}$ and $\boldsymbol{y}$ sharing a common element containing $a_{i}$,

$$
\left.|| \boldsymbol{x}\right|_{i}-|\boldsymbol{y}|_{i} \mid \leq 4
$$

Proof. Let $x$ be a common element of $\boldsymbol{x}$ and $\boldsymbol{y}$ containing $a_{i}$. Then by Lemma 4.22 we get

$$
\begin{aligned}
& \left.|| x\right|_{i}-|\boldsymbol{x}|_{i} \mid \leq 2, \\
& \left.|| x\right|_{i}-|\boldsymbol{y}|_{i} \mid \leq 2
\end{aligned}
$$

Combining the above inequalities proves the lemma. 


\section{The geometry of $\mathscr{E} S_{n}^{1}$}

5.1. Distance and $i$-length. We are now ready to estimate distances in $\varepsilon S_{n}^{1}$, based on how much $i$-length can change in a single proper nonempty index set.

Lemma 5.1. For any proper nonempty subset $S$ of the index set $\{1, \ldots, n\}$, any basis $\boldsymbol{x}=\left\{x_{1}, \ldots, x_{n}\right\}$ of $F_{n}$, and any $S$-transformation $\phi \in \operatorname{Aut}\left(F_{n}\right)$ which is the identity on $\boldsymbol{x}_{\bar{S}}$, we have that

$$
|x|_{i}-12 \leq|x \phi|_{i} \leq|x|_{i}+12 .
$$

Proof. If there exists some $x \in \boldsymbol{x}_{\bar{S}}$ such that $x$ contains an occurrence of $a_{i}^{ \pm 1}$, then since $x \in \boldsymbol{x} \cap \boldsymbol{x} \phi$, by Corollary 4.23

$$
\left.|| x\right|_{i}-|x \phi|_{i} \mid \leq 4
$$

and the lemma follows.

If no such $x$ exists, choose any $x \in \boldsymbol{x}_{\bar{S}}$ and let $y \in \boldsymbol{x}_{S}$ be an element of $\boldsymbol{x}$ which contains an occurrence of $a_{i}^{ \pm 1}$. Let $\boldsymbol{x}^{\prime}:=(\boldsymbol{x}-\{x\}) \cup\{x y\}$.

The bases $\boldsymbol{x}$ and $\boldsymbol{x}^{\prime}$ share $y$ in common, so by Corollary 4.23

$$
\left.|| \boldsymbol{x}\right|_{i}-\left|\boldsymbol{x}^{\prime}\right|_{i} \mid \leq 4
$$

Since $\boldsymbol{x}^{\prime}$ and $\boldsymbol{x}^{\prime} \phi$ share $x y$ in common, we get

$$
\left.|| \boldsymbol{x}^{\prime}\right|_{i}-\left|\boldsymbol{x}^{\prime} \phi\right|_{i} \mid \leq 4
$$

Also there must be an element among $z \in(\boldsymbol{x} \phi)_{S}$ containing an occurrence of $a_{i}$ (otherwise $\boldsymbol{x} \phi$ would not contain an occurrence of $a_{i}$ ). This element $z$ is common for bases $\boldsymbol{x} \phi$ and $\boldsymbol{x}^{\prime} \phi$ yielding

$$
\left.|| \boldsymbol{x}^{\prime} \phi\right|_{i}-|\boldsymbol{x} \phi|_{i} \mid \leq 4
$$

Finally, combining the inequalities (6), (7) and (8), we obtain the statement of the lemma.

It seems that, with more careful bookkeeping, the constant 12 might be able to be improved.

Corollary 5.2. Let $\boldsymbol{x}$ be a basis of $F_{n}$. Then the number of index changes in a decomposition into a product of $\mathcal{S}$-transformations of a transformation in $\operatorname{Aut}\left(F_{n}\right)$ taking $\boldsymbol{a}$ to $\boldsymbol{x}$ is bounded below by $\frac{1}{24}|\boldsymbol{x}|_{i}-1$.

Proof. For any index set $S$, an $S$-transformation can be written as a product of an $S$-transformation which is the identity on $\boldsymbol{x}_{S}$ and an $S$-transformation which is the identity on $\boldsymbol{x}_{\bar{S}}$. Applying Lemma 5.1 twice, we see an $S$-transformation can change $i$-length by at most 24 . The term " 1 " in the statement corresponds to the fact that a product of $k \delta$-transformations requires $k-1$ index changes. 
This corollary, combined with Theorem 3.2, shows our main computational theorem:

Theorem 5.3. Let $\boldsymbol{x}$ be a basis of $F_{n}$, expressed in terms of a fixed standard basis $\boldsymbol{a}$. For any index $i$ and any index sets $S_{\boldsymbol{a}}$ and $S_{\boldsymbol{x}}$,

$$
d_{\mathcal{E} S_{n}^{1}}\left(\left(\boldsymbol{a}, S_{\boldsymbol{a}}\right),\left(\boldsymbol{x}, S_{\boldsymbol{x}}\right)\right) \geq \frac{|\boldsymbol{x}|_{i}}{24}-1 .
$$

Proof. By Theorem 3.2, $d_{\mathcal{E} S_{n}^{1}}\left(\left(\boldsymbol{a}, S_{\boldsymbol{a}}\right),\left(\boldsymbol{x}, S_{\boldsymbol{x}}\right)\right)$ is greater than or equal to the number of compatible index changes required to get from $\left(\boldsymbol{a}, S_{\boldsymbol{a}}\right)$ to $\left(\boldsymbol{x}, S_{\boldsymbol{x}}\right)$, which, in turn, is not smaller than the number of arbitrary index changes. By Corollary 5.2 the last quantity is bounded below by $\frac{|\boldsymbol{x}|_{i}}{24}-1$.

5.2. $\mathcal{E} S_{n}^{1}$ is not hyperbolic. Corollary 5.2 is useful for estimating distances in $\mathcal{E} S_{n}^{1}$. For instance, we may now apply this corollary to show that $\mathcal{E} S_{n}^{1}$ is not hyperbolic in the sense of Gromov, by identifying quasiflats - that is, a quasiisometric embedding $\mathbb{R}^{k} \rightarrow \varepsilon S_{n}^{1}$ for $k>1$.

Let $p_{t}:=a_{1}^{t+1} a_{2}^{t+1} \cdots a_{n-1}^{t+1} a_{1}^{t+1} a_{2}^{t+1} a_{1}^{t+1}$. Note that for $t \geq 1$ the augmented Whitehead graph of $p_{t}$ looks similar to the graph shown in Figure 3, and removing vertices corresponding to $a_{n}$ and $a_{n}^{-1}$ will produce graphs without cut vertices. We propose to embed the integer lattice $\mathbb{Z}^{m}$ quasiisometrically into $\varepsilon S_{n}^{1}$ by the map $\psi$ which takes $\left(k_{1}, k_{2}, \ldots, k_{m}\right) \in \mathbb{Z}^{m}$ to the vertex $\psi\left(k_{1}, k_{2}, \ldots, k_{m}\right)=(\boldsymbol{x}, S)$ of $\mathcal{E} S_{n}^{1}$, where $S$ is an arbitrary proper nontrivial subset of $\{1,2, \ldots, n\}$ and $\boldsymbol{x}$ is obtained from the standard basis $\boldsymbol{a}$ by replacing $a_{n}$ by $a_{n} p_{1}^{k_{1}} p_{2}^{k_{2}} \cdots p_{m}^{k_{m}}$.

Theorem 5.4. The map $\psi$ yields an $m$-dimensional quasiflat in $\varepsilon \delta_{n}^{1}$.

Proof. To see that $\psi$ is indeed a quasiisometric embedding, consider the images of two points, $\left(k_{1}, k_{2}, \ldots, k_{m}\right)$ and $\left(l_{1}, l_{2}, \ldots, l_{m}\right)$ under $\psi$. In the domain, these points are of distance

$$
d=\sum_{t=1}^{m}\left|k_{t}-l_{t}\right|
$$

apart. In the codomain, the distance between $\psi\left(k_{1}, k_{2}, \ldots, k_{m}\right)$ and $\psi\left(l_{1}, l_{2}, \ldots, l_{m}\right)$ is the same as the distance between the basepoint $\boldsymbol{a}$ and the point represented by the standard basis with $a_{n}$ replaced by $a_{n} \omega$, where

$$
\omega=p_{m}^{-k_{m}} p_{m-1}^{-k_{m-1}} \cdots p_{1}^{-k_{1}} p_{1}^{l_{1}} p_{2}^{l_{2}} \cdots p_{m}^{l_{m}}
$$

after free reduction.

By Theorem 5.3 and the definition of full $i$-length, the latter distance is bounded below by

$$
\frac{1}{24}|\omega|_{n}^{\mathrm{cr}}-1
$$


We claim $|\omega|_{n}^{\text {cr }} \geq \frac{d}{11}-\frac{21}{11}$, as follows. By Lemma $4.11|\omega|_{n}^{\text {cr }}$ can be estimated from below by

$$
|\omega|_{n}^{\mathrm{cr}} \geq \min _{\mathcal{F} \in S}\left(\max \left\{\frac{|\mathcal{F}|}{2}-1, \frac{1}{5}|\omega-\mathscr{F}|_{n}^{\text {simple }}-3\right\}\right),
$$

where $S$ is the set consisting of all nested families of canceling pairs in $\omega$. Let $\mathcal{F}$ denote the family of canceling pairs in $\omega$ that minimizes the bound in (10).

There may be free cancellations of two types in $\omega$. First, several full occurrences of $p_{t}$ may cancel with full occurrences of $p_{t}^{-1}$ in the middle where $p_{1}^{-k_{1}}$ and $p_{1}^{l_{1}}$ meet, and second, there may be cancellation of two occurrences of $p_{t}$ for different $t$. In the second case, by the definition of $p_{t}$, the only part that may cancel is a subword of either the last and/or the first syllable of the form $a_{1}^{t+1}$. However, reductions of the second type preserve Whitehead graphs in the following sense: the Whitehead graph of the uncanceled subword $q$ of every copy of $p_{t}^{ \pm 1}$ (with vertices $a_{n}^{ \pm 1}$ removed) will still not have a cut vertex. We will call such a subword $q$ a leftover of type $t$ and denote by $q^{(t)}$. Each $q^{(t)}$ contains $a_{2}^{t+1} \ldots a_{n-1}^{t+1} a_{1}^{t+1} a_{2}^{t+1}$ as a subword.

Consider canceling pairs in $\mathcal{F}$. A lower bound for $|\omega-\mathcal{F}|_{n}^{\text {simple }}$ is the number of $q^{(t)}$ 's which do not intersect either element of any pair in $\mathscr{F}$, since each such $q^{(t)}$ contributes at least 1 to the sum in the definition of $|\omega-\mathscr{F}|_{n}^{\text {simple }}$. Therefore we just need to find a lower bound on the number of $\operatorname{such} q^{(t)}$ 's. Some occurrences of $q^{(t)}$ will be completely contained in an element of some canceling pair in $\mathcal{F}$ with an occurrence of $\left(q^{(t)}\right)^{-1}$ contained in the other element of the same pair. Throwing away all such occurrences, there are at least $\left|k_{t}-l_{t}\right|$ occurrences of $\left(q^{(t)}\right)^{ \pm 1}$ left for each $t$, and hence at least $d$ possible $q^{(t)}$ 's for $t$ arbitrary. For these remaining $q^{(t)}$ 's, by the definition of $p_{t}$, a given canceling pair may involve at most 4 different occurrences of $q^{(t)}$ 's for possibly different values of $t$. Indeed, each canceling pair involves two words, each of which can intersect some $q^{(t)}$ in an initial segment and/or a terminal segment. Each such segment can interfere with at most one occurrence of $q^{(t)}$. Thus, at most $4|\mathcal{F}|$ of the $d$ remaining $q^{(t)}$ 's are not disjoint from $\mathcal{F}$, and so at least $d-4|\widetilde{F}|$ occurrences of the $q^{(t)}$ 's do not intersect either element of any pair in $\mathcal{F}$.

Hence,

$$
\frac{1}{5}|\omega-\mathscr{F}|_{n}^{\text {simple }}-3 \geq \frac{1}{5}((d-4|\mathcal{F}|)+|\mathcal{F}|)-3=\frac{d}{5}-\frac{3}{5}|\mathcal{F}|-3 .
$$

From (10) we obtain

$$
|\omega|_{n}^{\mathrm{cr}} \geq \max \left\{\frac{|\mathcal{F}|}{2}-1, \frac{d}{5}-\frac{3}{5}|\mathcal{F}|-3\right\},
$$

If $|\mathcal{F}| \geq \frac{2}{11} d-\frac{20}{11}$ then

$$
|\omega|_{n}^{\mathrm{cr}} \geq \frac{|\mathcal{F}|}{2}-1 \geq \frac{d}{11}-\frac{21}{11} .
$$


But if $|\mathcal{F}|<\frac{2}{11} d-\frac{20}{11}$ then

$$
|\omega|_{n}^{\mathrm{cr}} \geq \frac{d}{5}-\frac{3}{5}|\mathcal{F}|-3>\frac{d}{11}-\frac{21}{11} .
$$

In either case, as claimed, $|\omega|_{n}^{\mathrm{cr}} \geq \frac{d}{11}-\frac{21}{11}$.

Combining this claim with the lower bound in (9), we have that the distance between the vertices $\psi\left(k_{1}, k_{2}, \ldots, k_{m}\right)$ and $\psi\left(l_{1}, l_{2}, \ldots, l_{m}\right)$ is bounded below by $\frac{d}{24 \cdot 11}-\frac{21}{24 \cdot 11}-1=\frac{1}{264} d-\frac{95}{88}$.

We claim the distance between $\psi\left(k_{1}, k_{2}, \ldots, k_{m}\right)$ and $\psi\left(l_{1}, l_{2}, \ldots, l_{m}\right)$ is also bounded above by $d+m$, as follows. Without loss of generality, fix $S=\{n\}$. Recall a vertex in $\mathscr{E} S_{n}^{1}$ is defined up to conjugation. Thus, for any word $w$,

$$
\left[\left\langle a_{1}, \ldots, a_{n-1}\right\rangle *\left\langle a_{n}\right\rangle\right]=\left[\left\langle a_{1}^{w}, \ldots, a_{n-1}^{w}\right\rangle *\left\langle a_{n}^{w}\right\rangle\right] .
$$

Thus, the following describes a path from $\psi\left(k_{1}, k_{2}, \ldots, k_{m}\right)$ to $\psi\left(l_{1}, l_{2}, \ldots, l_{m}\right)$ :

$$
\begin{aligned}
\psi\left(k_{1}, k_{2}, \ldots, k_{m}\right) & =\left[\left\langle a_{1}, \ldots, a_{n-1}\right\rangle *\left\langle a_{n} p_{1}^{k_{1}} p_{2}^{k_{2}} \ldots p_{m}^{k_{m}}\right\rangle\right] \\
& =\left[\left\langle a_{1}, \ldots, a_{n-1}\right\rangle *\left\langle p_{1}^{-k_{1}} \ldots p_{m}^{-k_{m}} a_{n}\right\rangle\right] \\
& \rightarrow\left[\left\langle a_{1}, \ldots, a_{n-1}\right\rangle *\left\langle p_{1}^{-k_{1}} \ldots p_{m}^{-k_{m}} a_{n} p_{1}^{l_{1}-k_{1}}\right\rangle\right] \\
& =\left[\left\langle a_{1}, \ldots, a_{n-1}\right\rangle *\left\langle p_{2}^{-k_{2}} \ldots p_{m}^{-k_{m}} a_{n} p_{1}^{l_{1}}\right\rangle\right] \\
& \rightarrow\left[\left\langle a_{1}, \ldots, a_{n-1}\right\rangle *\left\langle p_{2}^{-k_{2}} \ldots p_{m}^{-k_{m}} a_{n} p_{1}^{l_{1}} p_{2}^{l_{2}-k_{2}}\right\rangle\right] \\
& =\left[\left\langle a_{1}, \ldots, a_{n-1}\right\rangle *\left\langle p_{3}^{-k_{3}} \ldots p_{m}^{-k_{m}} a_{n} p_{1}^{l_{1}} p_{2}^{l_{2}}\right\rangle\right] \\
& \rightarrow \cdots \\
& \rightarrow\left[\left\langle a_{1}, \ldots, a_{n-1}\right\rangle *\left\langle p_{m}^{-k_{m}} a_{n} p_{1}^{l_{1}} p_{2}^{l_{2}} \ldots p_{m-1}^{l_{m}-1} p_{m}^{l_{m}-k_{m}}\right\rangle\right] \\
& =\left[\left\langle a_{1}, \ldots, a_{n-1}\right\rangle *\left\langle a_{n} p_{1}^{l_{1}} p_{2}^{l_{2}} \ldots p_{m-1}^{l_{m-1}} p_{m}^{l_{m}}\right\rangle\right] \\
& =\psi\left(l_{1}, l_{2}, \ldots, l_{m}\right) .
\end{aligned}
$$

At each arrow, the above sequence is the same: for some integers $i$ and $j$, we append $p_{i}^{j}$ to the last factor in a vertex of the form $\left[\left\langle a_{1}, \ldots, a_{n-1}\right\rangle *\langle u a v\rangle\right]$. We claim this may be done in via at most $2 j+1$ steps in $\varepsilon S_{n}^{1}$, by the following edge path, where in this sequence, arrows each represent crossing exactly 1 edge of $\& \delta_{n}^{1}$ :

$$
\begin{aligned}
& {\left[\left\langle a_{1}, \ldots, a_{n-1}\right\rangle *\langle u a v\rangle\right]} \\
& \quad \rightarrow\left[\left\langle a_{n-1}\right\rangle *\left\langle a_{1}, \ldots, a_{n-2}, u a_{n} v\right\rangle\right] \\
& \quad=\left[\left\langle a_{n-1}\right\rangle *\left\langle a_{1}, \ldots, a_{n-2}, u a_{n} v a_{1}^{i+1} a_{2}^{i+1} \cdots a_{n-2}^{i+1}\right\rangle\right]
\end{aligned}
$$




$$
\begin{aligned}
& \rightarrow\left[\left\langle a_{3}, \ldots, a_{n-2}\right\rangle *\left\langle a_{1}, a_{2}, a_{n-1}, u a_{n} v a_{1}^{i+1} a_{2}^{i+1} \cdots a_{n-2}^{i+1}\right\rangle\right] \\
& =\left[\left\langle a_{3}, \ldots, a_{n-2}\right\rangle *\left\langle a_{1}, a_{2}, a_{n-1}, u a_{n} v p_{i}\right\rangle\right] \\
& \rightarrow\left[\left\langle a_{n-1}\right\rangle *\left\langle a_{1}, \ldots, a_{n-2}, u a_{n} v p_{i}\right\rangle\right] \\
& =\left[\left\langle a_{n-1}\right\rangle *\left\langle a_{1}, \ldots, a_{n-2}, u a_{n} v p_{i} a_{1}^{i+1} a_{2}^{i+1} \cdots a_{n-2}^{i+1}\right\rangle\right] \\
& \rightarrow\left[\left\langle a_{3}, \ldots, a_{n-2}\right\rangle *\left\langle a_{1}, a_{2}, a_{n-1}, u a_{n} v p_{i} a_{1}^{i+1} a_{2}^{i+1} \cdots a_{n-2}^{i+1}\right\rangle\right] \\
& =\left[\left\langle a_{3}, \ldots, a_{n-2}\right\rangle *\left\langle a_{1}, a_{2}, a_{n-1}, u a_{n} v p_{i}^{2}\right\rangle\right] \\
& \rightarrow \cdots \\
& =\left[\left\langle a_{3}, \ldots, a_{n-2}\right\rangle *\left\langle a_{1}, a_{2}, a_{n-1}, u a_{n} v p_{i}^{j}\right\rangle\right] \\
& \rightarrow\left[\left\langle a_{1}, \ldots, a_{n-1}\right\rangle *\left\langle u a v p_{i}^{j}\right\rangle\right]
\end{aligned}
$$

Note here we shown the path when $j>0$; the path when $j<0$ is similar. Combining these two descriptions, we have that:

$$
d_{\mathcal{E} S_{n}^{1}}\left(\psi\left(k_{1}, k_{2}, \ldots, k_{m}\right), \psi\left(l_{1}, l_{2}, \ldots, l_{m}\right)\right) \leq \sum_{i=1}^{m} 2\left|l_{i}-k_{i}\right|+1=2 d+m .
$$

As distances are bounded both above and below, we have a quasiisometric embedding.

As immediate corollaries, we obtain:

Corollary 5.5. The graph $\mathcal{E} S_{n}^{1}$ is not hyperbolic in the sense of Gromov.

This shows that $\varepsilon S_{n}^{1}$ does not have the hyperbolicity desired for an analogue for $\operatorname{Out}\left(F_{n}\right)$ of the curve complex for the mapping class group. The hyperbolicity of the curve complex was shown by Masur and Minsky [25], and has proven to be useful in numerous situations.

Corollary 5.6. The space $\mathcal{E} S_{n}^{1}$ has infinite asymptotic dimension. The dimension of every asymptotic cone of $\& \delta_{n}^{1}$ is infinite.

Corollary 5.7. The identity map on vertices between $\& S_{n}^{1}$ and $\mathcal{F F}_{\mathcal{F}_{n}}^{1}$ is not a quasiisometry. Moreover, there is no coarsely $\operatorname{Out}\left(F_{n}\right)$-equivariant quasiisometry between $\varepsilon \delta_{n}^{1}$ and $\mathcal{F F}_{n}^{1}$.

Proof. The first half follows immediately from Theorem 5.4, as the set $\psi \mathbb{Z}^{m} \subset \varepsilon \delta_{n}^{1}$ has diameter 1 in $\mathcal{F}_{\mathcal{F}^{1}}{ }_{n}$ : for $k \neq n$, the element $a_{k}$ has translation length 0 on the Bass-Serre tree of every element in $\psi \mathbb{Z}^{m}$.

For the second half we note that since $\operatorname{Out}\left(F_{n}\right)$ acts on both $\mathcal{E} S_{n}^{1}$ (and $\mathcal{F}_{\mathcal{F}}{ }_{n}^{1}$ ) by isometries, for each $\phi \in \operatorname{Out}\left(F_{n}\right)$ the orbits under powers of $\phi$ of vertices of $\varepsilon S_{n}^{1}$ (and 
$\left.\mathcal{F} \mathcal{F}_{n}^{1}\right)$ are either all bounded or all unbounded. Now consider $\phi \in \operatorname{Out}\left(F_{n}\right)$ taking the standard basis $\boldsymbol{a}$ of $F_{n}$ to the basis obtained from $\boldsymbol{a}$ by replacing $a_{n}$ with $a_{n} p_{1}$. By construction, for each index set $S$ the orbit of $(\boldsymbol{a}, S)$ in $\varepsilon S_{n}^{1}$ under iterations of $\phi$ is unbounded as the $i$-length of $\phi^{n}(\boldsymbol{a})$ grows. But the same orbit is bounded in $\mathscr{F} \mathcal{F}_{n}{ }_{n}^{1}$. Thus, there is no coarsely $\operatorname{Out}\left(F_{n}\right)$-equivariant quasiisometry between $\mathscr{E} \delta_{n}^{1}$ and $\mathscr{F}_{\mathcal{F}}{ }_{n}^{1}$ because every orbit in $\mathscr{F}_{\mathcal{F}}{ }_{n}^{1}$ under iterations of $\phi$ is bounded and cannot be an image of an unbounded orbit in $\& S_{n}^{1}$.

An analogous results with identical proofs hold true for the relationships between the free factorization graph $\& S_{n}^{1}$ and the free factor graph $\mathcal{F}_{n}^{1}$ and between $\& S_{n}^{1}$ and the free splitting graph $\mathscr{F} S_{n}^{1}$. There is a natural (coarsely well defined for $n>2$ ) map $\Sigma: \mathcal{E} S_{n}^{1} \rightarrow \mathscr{F}_{n}^{1}$ defined by sending a vertex $[A * B]$ in $\mathcal{E} S_{n}^{1}$ to the vertex $[A]$ in $\widetilde{F}_{n}{ }_{n}$. This map is induced by the same map on vertices from $\mathscr{F}_{\mathcal{F}}{ }_{n}^{1}$ to $\mathscr{F}_{n}^{1}$, which is a coarsely $\operatorname{Out}\left(F_{n}\right)$-equivariant quasiisometry. Also there is a natural embedding $\imath: \mathcal{E} S_{n}^{1} \rightarrow \mathcal{F} S_{n}^{1}$ defined by sending a vertex $[A * B]$ in $\mathcal{E} S_{n}^{1}$ to the vertex $[A * B]$ in $\mathscr{F} S_{n}^{1}$, which is quasisurjection. However, neither of the above maps is a quasiisometry.

Corollary 5.8. The maps $\Sigma: \mathcal{E} S_{n}^{1} \rightarrow \mathscr{F}_{n}^{1}$ and $\imath: \mathcal{E} S_{n}^{1} \rightarrow \mathscr{F}_{n}^{1}$ are not quasiisometries. Moreover, there is no coarsely $\operatorname{Out}\left(F_{n}\right)$-equivariant quasiisometry between $\mathcal{E} S_{n}^{1}$ and $\mathcal{F}_{n}^{1}$, and between $\mathcal{E} S_{n}^{1}$ and $\mathcal{F}_{S_{n}}^{1}$.

The last corollary provides a negative answer to a question of Bestvina and Feighn (the first half of Question 4.4 in [6]).

Finally, we mention that the recent results of Bestvina and Feighn [7] and of Handel and Mosher [14] claiming hyperbolicity of $\mathscr{F}_{n}^{1}$ and $\mathscr{F} S_{n}^{1}$ respectively, can be used to generalize Corollaries 5.7 and 5.8.

Corollary 5.9. The edge splitting graph $\mathcal{E} S_{n}^{1}$ is not quasiisometric to any of $\mathcal{F} \mathcal{F}_{n}{ }_{n}$, $\widetilde{F}_{n}^{1}$ and $\widetilde{F}_{{ }_{n}}^{1}$.

\section{References}

[1] J.Aramayona and J. Souto, Automorphisms of the graph of free splittings. Michigan Math. J. 60 (2011), no. 3, 483-493. Zbl https://zbmath.org/?q=an:1242.05117 MR 2861084

[2] J. Behrstock, M. Bestvina, and M. Clay, Growth of intersection numbers for free group automorphisms. J. Topol. 3 (2010), no. 2, 280-310. Zbl 1209.20031 MR 2651361

[3] J. Behrstock, B. Kleiner, Y. Minsky, and L. Mosher, Geometry and rigidity of mapping class groups. Geom. Topol. 16 (2012), no. 2, 781-888. Zbl 1281.20045 MR 2928983

[4] Y. Berchenko-Kogan, Distance in the ellipticity graph. Preprint, arXiv:1006.4853.

[5] M. Bestvina, K. Bromberg, and F. Koji, Constructing group actions on quasi-trees and applications to mapping class groups Preprint, arXiv:1006.1939. 
[6] M. Bestvina and M. Feighn, A hyperbolic Out $\left(F_{n}\right)$-complex. Groups Geom. Dyn. 4 (2010), no. 1, 31-58. Zbl 1190.20017 MR 2566300

[7] M. Bestvina and M. Feighn, Hyperbolicity of the complex of free factors. Adv. Math. 256 (2014), 104-155; Corrigendum ibid. 259 (2014), 843. Zbl 06284922 MR 3177291

[8] F. Bonahon, Geodesic currents on negatively curved groups. In Arboreal group theory (Berkeley, CA, 1988), Math. Sci. Res. Inst. Publ. 19, Springer, New York 1991, 143-168. Zbl 0772.57004 MR 1105332

[9] M. Culler and K. Vogtmann, Moduli of graphs and automorphisms of free groups. Invent. Math. 84 (1986), no. 1, 91-119. Zbl https://zbmath.org/?q=an:0589.20022 MR 0830040

[10] Ma. Day and A. Putman, The complex of partial bases for $F_{n}$ and finite generation of the Torelli subgroup of Aut $\left(F_{n}\right)$. Geom. Dedicata 164 (2013), 139-153. Zbl 06171542 MR 3054621

[11] A. Dranishnikov and M. Sapir, On the dimension growth of groups. J. Algebra 347 (2011), 23-39; Corrigendum ibid. 370 (2012), 407-409. Zbl 1277.20051 MR 2846394

[12] D. Farley, Homology of tree braid groups. In Topological and asymptotic aspects of group theory, Contemp. Math. 394k, Amer. Math. Soc., Providence, RI, 2006, 101-112. Zbl 1102.20028 MR 2216709

[13] V. Guirardel, Cœur et nombre d'intersection pour les actions de groupes sur les arbres. Ann. Sci. École Norm. Sup. (4) 38 (2005), no. 6, 847-888. Zbl 1110.20019 MR 2216833

[14] M. Handel and L. Mosher, The free splitting complex of a free group I: hyperbolicity. Geom. Topol. 17 (2013), no. 3, 1581-1672. Zbl 1278.20053 MR 3073931

[15] A. Hatcher, Homological stability for automorphism groups of free groups. Comment. Math. Helv. 70 (1995), no. 1. 39-62. Zbl 0836.57003 MR 1314940

[16] A. Hatcher and K. Vogtmann, Cerf theory for graphs. J. London Math. Soc. (2) 58 (1998), no. 3, 633-655. Zbl 0922.57001 MR 1678155

[17] A. Hatcher and K. Vogtmann, The complex of free factors of a free group. Quart. J. Math. Oxford Ser. (2) 49 (196) (1998), 459-468. Zbl 0935.20015 MR 1660045

[18] L. Ji, Asymptotic dimension and the integral $K$-theoretic Novikov conjecture for arithmetic groups. J. Differential Geom. 68 (2004), no. 3, 535-544. Zbl 1079.55012 MR 2144540

[19] I. Kapovich, Currents on free groups. In Topological and asymptotic aspects of group theory, Contemp. Math. 394, Amer. Math. Soc., Providence, RI, 2006, 149-176. Zbl 1110.20034 MR 2216713

[20] I. Kapovich and M. Lustig, Geometric intersection number and analogues of the curve complex for free groups. Geom. Topol. 13 (2009), no. 3, 1805-1833. Zbl 1194.20046 MR 2496058

[21] I. Kapovich and A. Myasnikov, Stallings foldings and subgroups of free groups. J. Algebra 248 (2002), 608-668. Zbl 1001.20015 MR 1882114

[22] M. Lustig, A generalized intersection form for free groups. Preprint, 2004.

[23] R. C. Lyndon and P. E. Schupp, Combinatorial group theory. Classics Math,, SpringerVerlag, Berlin 2001. Reprint of the 1977 edition. Zbl 0997.20037 MR 1812024 
[24] W. Magnus, A. Karrass, and D. Solitar, Combinatorial group theory. Reprint of the 1976 second edition, Dover Publications Inc., Mineola, NY, 2004. Zbl 1130.20307 MR 2109550

[25] H. A. Masur and Y. N. Minsky, Geometry of the complex of curves. I. Hyperbolicity. Invent. Math. 138 (1999), no. 1, 103-149. Zbl 0941.32012 MR 1714338

[26] J. Nielsen, Die Isomorphismengruppe der freien Gruppen. Math. Ann. 91 (1924), no. 3-4, 169-209. JFM 50.0078.04 MR 1512188

[27] S. Schleimer, Notes on the complex of curves. Preprint 2006, available at http://www.warwick.ac.uk/ masgar/Maths/notes.pdf. Zbl MR

[28] J. R. Stallings, Whitehead graphs on handlebodies. In Geometric group theory down under (Canberra, 1996), de Gruyter, Berlin 1999, 317-330. Zbl 1127.57300 MR 1714852

[29] K. Vogtmann, Automorphisms of free groups and outer space. In Proceedings of the Conference on Geometric and Combinatorial Group Theory, Part I (Haifa, 2000), Geom. Dedicata 94 2002, 1-31. Zbl 1017.20035 MR 1950871

[30] J. H. C. Whitehead, On certain sets of elements in a free group. Proc. London Math. Soc. 41 (1936), 48-56. JFM 62.0079.04 MR 1575455

Received June 13, 2012; revised September 17, 2012

L. Sabalka, Department of Mathematics and Computer Science, Saint Louis University, St Louis, MO 63112, U.S.A.

E-mail: 1sabalka@slu.edu

D. Savchuk, Department of Mathematics and Statistics, University of South Florida, Tampa, FL 33620, U.S.A.

E-mail: savchuk@usf.edu 